\title{
Quantum-optical magnets with competing short- and long-range interactions: Rydberg-dressed spin lattice in an optical cavity
}

\author{
Jan Gelhausen, ${ }^{*}$ Michael Buchhold, Achim Rosch, and Philipp Strack ${ }^{\dagger}$ \\ Institut für Theoretische Physik, Universität zu Köln, D-50937 Cologne, Germany
}

(Dated: August 19, 2021)

\begin{abstract}
The fields of quantum simulation with cold atoms [1] and quantum optics [2] are currently being merged. In a set of recent pathbreaking experiments with atoms in optical cavities [3, 4], lattice quantum many-body systems with both, a short-range interaction and a strong interaction potential of infinite range -mediated by a quantized optical light field- were realized. A theoretical modelling of these systems faces considerable complexity at the interface of: (i) spontaneous symmetry-breaking and emergent phases of interacting many-body systems with a large number of atoms $N \rightarrow \infty$, (ii) quantum optics and the dynamics of fluctuating light fields, and (iii) non-equilibrium physics of driven, open quantum systems. Here we propose what is possibly the simplest, quantum-optical magnet with competing short- and long-range interactions, in which all three elements can be analyzed comprehensively: a Rydberg-dressed spin lattice [5] coherently coupled to a single photon mode. Solving a set of coupled even-odd sublattice master equations for atomic spin and photon mean-field amplitudes, we find three key results. (R1): Superradiance and a coherent photon field can coexist with spontaneously broken magnetic translation symmetry. The latter is induced by the short-range nearest-neighbor interaction from weakly admixed Rydberg levels. (R2): This broken even-odd sublattice symmetry leaves its imprint in the light via a novel peak in the cavity spectrum beyond the conventional polariton modes. (R3): The combined effect of atomic spontaneous emission, drive, and interactions can lead to phases with anomalous photon number oscillations. Extensions of our work include nano-photonic crystals coupled to interacting atoms and multi-mode photon dynamics in Rydberg systems.
\end{abstract}

\footnotetext{
*jg@thp.uni-koeln.de

† strack@thp.uni-koeln.de; http://www.thp.uni-koeln.de/ strack/
} 


\section{CONTENTS}

I. Introduction and key results 3

A. Model: Rydberg-dressed spin lattice coupled to single-mode optical light field 4

B. Result 1: Coexistence of superradiance and magnetic translation symmetry-breaking 6

C. Result 2: Even-odd sublattice peak in cavity spectrum 9

$\begin{array}{ll}\text { D. Result 3: Photon number oscillations } & 10\end{array}$

II. Ideas for quantum-optical implementation of the model 13

III. Coupled even-odd sublattice mean-field master equations for atoms and photons 16

IV. Derivation and detailed discussion of results 17

A. Result 1: Coexistence of superradiance and magnetic translation symmetry-breaking 17

1. Phase boundaries and order parameters with photon losses $(\kappa \neq 0, \gamma=0)$

B. Result 2: Even-odd sublattice peak in cavity spectrum 21

1. Derivation of the cavity output spectrum $(\kappa \neq 0, \gamma=0)$

2. Low-frequency pole structure for the case $(\kappa \neq 0, \gamma=0)$

C. Result 3: Photon number oscillations 25

1. Phase boundaries and order parameters with spontaneous emission $(\kappa \neq 0, \gamma \neq 0) \quad 25$

$\begin{array}{lr}\text { V. Conclusions and future directions } & 28\end{array}$

$\begin{array}{ll}\text { Acknowledgments } & 29\end{array}$

A. Mean-field solution of the $\mathrm{T}=0$ equilibrium spin model 30

B. Transformation of fully time-dependent model into rotating frame 31

$\begin{array}{ll}\text { References } & 35\end{array}$ 


\section{INTRODUCTION AND KEY RESULTS}

The fields of quantum simulation with cold atoms [1] and quantum optics [2] are currently being merged. On the one hand, to coherently couple photons to low-entropy, correlated quantum many-body states - the objects of desire of quantum simulators- offers new possibilities to imprint atomic coherences and quantum correlations onto quantum light such as for example dissipatively anti-bunched photons [6] or a "many-fermion" EIT [7] window without light absorption [8]. On the other hand, the inter-atomic interactions mediated by the photons opens up explorations of previously inaccessible phases such as long-ranged quantum spin- and charge glasses [9-13], bond-ordered phases [14], dynamical spin-orbit couplings [15, 16], or topological states carrying perpetual currents with dynamical gauge couplings [17, 18].

A major objective in the field of quantum simulation is to prepare and probe low-entropy quantum magnets. Existing efforts have focussed on magnetic interactions via superexchange [19], mapping to charge degrees of freedom [20], dipolar interactions [21] with polar molecules [22], magnetic atoms [23], and laser-dressed Rydberg atoms [5]. More complex interaction potentials necessary for frustrated magnetism have also been proposed [24-26]; optical pumping schemes should allow to access non-equilibrium magnets, too [27, 28]. One common goal of these efforts is the engineering of a single magnetic interaction rate with a certain angle-dependence and range, which can compete with kinetic energies, longitudinal fields, and the decay processes.

In this article, we want to initiate the study of quantum-optical magnets with competing short-and long-range interactions, the latter being mediated by a dynamical photon field. One may wonder how a single spin or quantum dipole (with in principle fixed charge distribution) can interact via two, competing interactions potentials with drastically different range and independently tuneable magnitude: At the core of our proposal is an atomic "two dipoles in-one" unit (illustrated below in Fig. 7), to which -depending on the principal quantum number and electronic transitions usedtwo different force-mediating photon fields can couple simultaneously (described in Sec. II).

In addition to the novel magnetic phases an array of such "two dipoles in-one" can attain, a key question is how the quantum dynamics of the photon field is affected by the magnetic correlations. Using an optical cavity for one of the photonic force carriers and a deep optical lattice to freeze out the motion of the atoms is a natural experimental set-up, which will allow non-destructive detection of purely magnetic correlations via the cavity output spectrum [3, 4, 29]. Let us note that the question of how quantum light interacts with a self-interacting set of qubits is of broader 
relevance including for example cavity Rydberg polaritons [30, 31], Rydberg-EIT setups [6, 32, 33] and nano-photonic devices[34-36]. Especially in reduced dimensions with confined electric fields, even small qubit-qubit interactions can have a huge effect.

These systems generate a lot of complexity at the interface of three typically only weakly connected areas of physics: (i) emergent phases and spontaneous symmetry-breaking of interacting many-body systems in the thermodynamic limit ( $N \rightarrow \infty$ number of qubits) (ii) quantum optics and the dynamics of fluctuating light fields (from $M=1$ to $M=\infty$ photon modes), and (iii) non-equilibrium physics of driven, open quantum systems, due to drive and multiple loss channels such as photon decay with rate $\kappa$ and atomic spontaneous emission with rate $\gamma$.

The goal of the present paper is to provide a "base case" or the simplest prototype of a quantumoptical magnet with competing short- and long-range interactions, in which the interplay of the above mentioned (i)-(iii) can be transparently studied.

\section{A. Model: Rydberg-dressed spin lattice coupled to single-mode optical light field}

As a suitable model (Fig. 1), we propose to supplement the existing experimental set-ups [3, 4] by weakly admixing a Rydberg-level with relatively low principal quantum number $(n \sim 30)$. The other, but equivalent, point of view is to couple a Rydberg-dressed spin lattice [5] to a single mode of an optical resonator. See also Refs. 37 for a related setup but without a lattice. As we derive below in Sec. II, the pure spin-part of the Hamiltonian $H=H_{\text {spin }}+H_{\text {spin-light }}$ is

$$
H_{\text {spin }}=-\frac{\Delta}{2} \sum_{\ell=1}^{N} \sigma_{\ell}^{z}+\frac{V}{d} \sum_{\langle\ell m\rangle}\left(\frac{1+\sigma^{z}}{2}\right)_{\ell}\left(\frac{1+\sigma^{z}}{2}\right)_{m},
$$

where the sum $\sum_{\langle\ell m\rangle}$ goes over all nearest-neighbor pairs of the square lattice and $d=z / 2$ is the dimension of the lattice with $z$ the coordination number. For a negative $(-\Delta<0)$ longitudinal field it is favorable for the spins to point up along the $z$-axis $|\uparrow\rangle$. Competing against this is the repulsive or antiferromagnetic "Rydberg-mediated" term, which minimizes energy by pushing the spin in spatially alternating configurations, e. g. $|\uparrow \downarrow \uparrow \downarrow \ldots\rangle$. In contrast to a conventional Ising $\sim \sigma_{\ell}^{z} \sigma_{m}^{z}$ interaction term, the Rydberg interaction is conditioned on population in the upper state.

Non-trivial quantum fluctuations are added to $H_{\text {spin }}$ by coherent conversion of spin excitations into photons with rate $g$

$$
H_{\text {spin-light }}=\frac{g}{\sqrt{N}}\left(a+a^{\dagger}\right) \sum_{\ell=1}^{N}\left(\sigma_{\ell}^{+}+\sigma_{\ell}^{-}\right)+\omega_{0} a^{\dagger} a,
$$




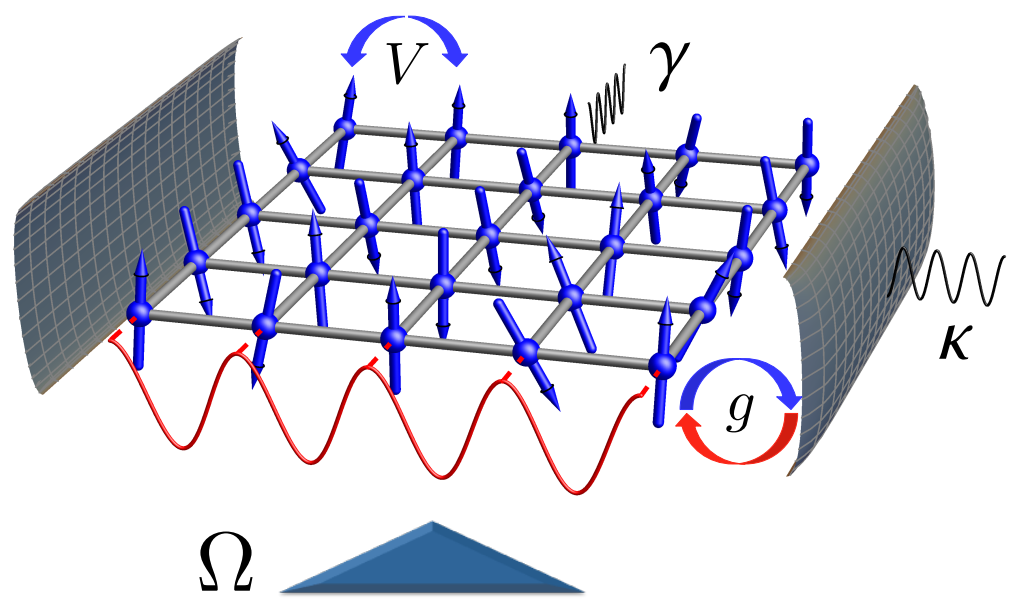

Figure 1. Rydberg-dressed spin lattice coupled to single-mode optical light field. We take the atoms to be in a deep optical lattice (for example a Mott insulator at unit filling) such that no motion occurs and only internal spin excitations drive the dynamics. From weakly admixing a Rydberg level, an effective nearestneighbor interaction (repulsive) $V$ competes with an effectively infinite range interaction from the single light mode of the cavity that couples all atoms with strength $g$. The atoms can spontaneously decay with rate $\gamma$ and photons can leave the system through the cavity mirrors with rate $\kappa$. The system is driven from the side with a laser of Rabi frequency $\Omega$ to deposit excitations into the system. Driven dipoles in other lattice geometries are also interesting to consider [38].

where $\omega_{0}$ is the effective cavity frequency in a rotating frame and $N$ is the number of atoms; the rescaling of the effective spin-light coupling with $1 / \sqrt{N}$ ensures a non-trivial thermodynamic limit (i.e. taking the system size and number of atoms to infinity keeping the atomic density and electric field strength per volume constant). All coupling constants appearing here are expressed in terms of fundamental quantum-optical parameters in Sec. II and $g$ is proportional to the external laser drive $\Omega$. Note that the $\left(a+a^{\dagger}\right)$ may be viewed to act on the spins like a "transverse field" in $x$-direction, whose value depends on the quantum state of the photons. The two states captured within the mean-field theory we use later are the cavity vacuum, that is, the Fock state with zero photon excitations: $a|0\rangle=0$. Then, $\left\langle a+a^{\dagger}\right\rangle=0$ and the spins see zero transverse field. The other option is the photon forming a coherent state in which $\left\langle a+a^{\dagger}\right\rangle \neq 0$; this is how we detect the superradiant phase below.

Our model is completed by the inclusion of Lindblad operators for photon losses through the mirrors with rate $\kappa$ and spontaneous emission of the atoms with rate $\gamma$ into the reservoir modes of 
the electromagnetic vacuum surrounding the cavity:

$$
\begin{aligned}
& \mathcal{L}_{\gamma}[\rho]=\frac{\gamma}{2} \sum_{\ell=1}^{N}\left[2 \sigma_{\ell}^{-} \rho \sigma_{\ell}^{+}-\left\{\sigma_{\ell}^{+} \sigma_{\ell}^{-}, \rho\right\}\right], \\
& \mathcal{L}_{\kappa}[\rho]=\kappa\left[2 a \rho a^{\dagger}-\left\{a^{\dagger} a, \rho\right\}\right],
\end{aligned}
$$

where $\rho$ is the system density matrix. Spatially modulated phases in the presence of coherent driving of lattice atoms have been discussed in an open, non-equilibrium setting in particular by Lee and collaborators [27, 28]; see also Ref. 39. Here, we extend such models by coupling the spin degrees of freedom to a quantum light field, which can also be in a zero-photon vacuum state with undetermined phase. In fact, the loss rate for the photons $\kappa$ wants to drive the photons into this vacuum state (i.e. the empty cavity $|0\rangle$ ).

We now present our main results from an analysis of Eqs. (1-4) using even-odd sublattice meanfield master equations (derived in Sec. III) and the input-output formalism. A detailed discussion and derivation of these results can be found in Sec. IV.

\section{B. Result 1: Coexistence of superradiance and magnetic translation symmetry-breaking}

Our first key result is Fig. 2: the non-equilibrium steady-stase phase diagram of Eqs. (1-4) setting the atomic spontaneous emission $\gamma$ to zero for now. Using cavity-assisted Raman transitions [40] to tune the atom-light coupling, this describes the limit of relatively far detuned excited states, where population in the decaying levels is suppressed. These phase diagrams are computed from solving for steady states of mean-field master equations for the real-valued atomic variables $\left(\left\langle\sigma^{x}\right\rangle,\left\langle\sigma^{y}\right\rangle,\left\langle\sigma^{z}\right\rangle\right)$ and the complex-valued photon expectation values $\left(\langle a\rangle,\left\langle a^{\dagger}\right\rangle\right)$, see Eqs. (12-15). This way of solving the problem implicitly takes first the thermodynamic limit $N \rightarrow \infty$ and subsequently the long-time limit $t \rightarrow \infty$. We keep $\kappa$ finite to account for photon losses. In App. A, we show how the somewhat unphysical limit $\kappa \rightarrow 0$ reproduces in fact the phase boundaries of a corresponding ground state $T=0$ model. In a quantum optics experiment, this most closely seems to correspond to the preparation protocol in which the interacting spins are prepared in a low-entropy state in a given phase, first without any coupling to the cavity (i.e. the transversal laser drive $\Omega$ turned off). Starting in the AFM phase in Fig. 2, for example, the coupling $g$ is then

ramped up and induces superradiance and the coexistence phase $A F M+S R$. In principle, the ramp of $g$ should be sufficiently slow not to de-equilibrate the AFM state but fast enough compared 


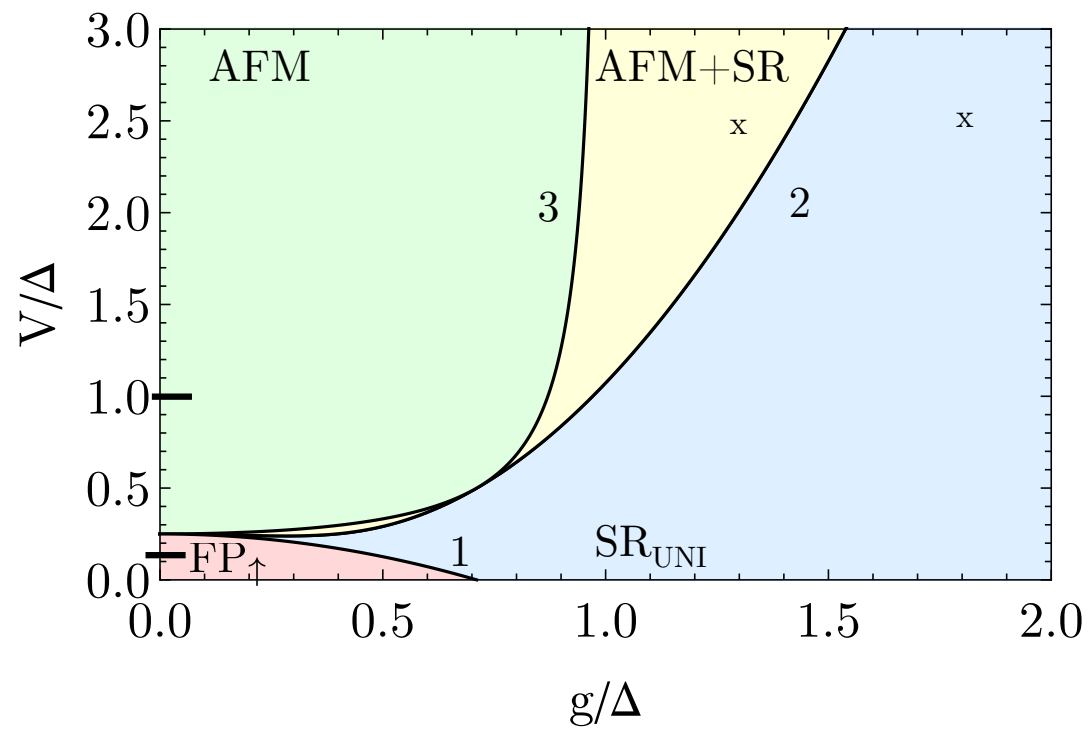

Figure 2. Non-equilibrium mean-field phase diagram of a Rydberg-dressed spin lattice (with nearest neighbor interaction $V$ ) coupled to a single-mode optical light field with rate $g$. In units of the spin longitudinal field $\Delta$. The key feature is the yellow strip, AFM+SR phase, in which spatially modulated magnetic moments coexist with a superradiant photon condensate, see Tab. I. This phase may be regarded as the magnetic analogue of the (superradiant) supersolid of moving lattice bosons in an optical cavity [3, 41-43]. The yellow strip merges into the $V$-axis at a multi-critical point from which four different phases can be reached by infinitesimal variation of parameters. At the multi-critical point $(g / \Delta=0, V / \Delta=1 / 4)$ the spins are "maximally soft", i. e., they feel zero effective, longitudinal field. Here this infinitesimal sensitivity to ordering is not rounded off by cavity decay induced noise. Recall that typically the cavity-induced decay rate for an atom is $\sim g^{2} \kappa /\left(\omega_{0}^{2}+\kappa^{2}\right)$. Here however the noise kicks of the two photon absorption and emission pathways destructively interfere in the level scheme Fig. 7, which, in turn, manifests itself in the $\sim g\left(\sigma_{\ell}^{+}+\sigma_{\ell}^{-}\right)\left(a+a^{\dagger}\right)$ coupling. $\mathrm{SR}_{\mathrm{UNI}}$ is a uniform superradiant phase in which the spins also develop an expectation value in $x$-direction. AFM stands for antiferromagnetic with differing magnetic moments on the even and the odd sublattice. $\mathrm{FP}_{\uparrow}$ is a fully polarized phase in which all spins point up. The magnetisations and the value of the photon condensate across the transitions are continuous. Cavity spectra at positions labeled with (x) are depicted in Fig. 9. Numerical parameters used: $\omega_{0} / \Delta=2.0, \kappa / \Delta=0.2$.

to $1 / \kappa$ in order not to loose excitations before they can interact and mediate the spin interaction. However, existing experiments and in particular the onset of superradiance are suprisingly robust against variations in the preparation scheme [3, 4, 44]. 


\begin{tabular}{c|c|c} 
Phase & Broken Symmetry & Order Parameter \\
\hline SR $_{\text {UNI }}$ & Superradiance $\mathbb{Z}_{2}$ & $\langle a\rangle \neq 0$ \\
AFM & Lattice translations $T_{\text {lat }}$ & $\left\langle\sigma_{e}^{z}\right\rangle-\left\langle\sigma_{0}^{z}\right\rangle \neq 0$ \\
AFM+SR & $\mathbb{Z}_{2}$ and $T_{\text {lat }}$ & $\left\langle\sigma_{e}^{z}\right\rangle-\left\langle\sigma_{0}^{z}\right\rangle \neq 0,\langle a\rangle \neq 0$ \\
FP & None & None
\end{tabular}

Table I. Order parameters for the phases in Fig. 2. Whenever the photon parity is broken, the $x$-projections of the spins also attain a finite expectation value $\left\langle\sigma^{x}\right\rangle \neq 0$.

The phases shown in Fig. 2 can be classified according to their "order parameters" in Table I. Let us describe the phases in more detail. Upon increasing the coupling to the photons along the $g$-axis in Fig. 2, for $V /|\Delta|<1 / 4$, a fully polarized phase $\left(\mathrm{FP}_{\uparrow},|\uparrow \uparrow \ldots\rangle\right)$ becomes superradiant crossing the Dicke transition, which has been studied in detail for both, the closed thermal and ground states as well as the open variant (most recently including also single-site atomic spontaneous emission $[45,46])$. The symmetry, which is spontaneously broken is

$$
\mathbb{Z}_{2}:\left[a+a^{\dagger}, \sigma_{\ell}^{x}, \sigma_{\ell}^{y}\right] \rightarrow\left[-\left(a+a^{\dagger}\right),-\sigma_{\ell}^{x},-\sigma_{\ell}^{y}\right]
$$

The experimental signature is a jump of the photon number inside the resonator [3,45]. Recall that here we have the atomic spins pinned in an optical lattice, which takes away the photon recoil momenta transferred from the photons to the atoms. This is in contrast to the realizations of the Dicke model with momentum states of the atomic gas [44, 47, 48]; therein the onset of superradiance is accompanied by even-odd checkerboard formation. Here, with the setup Fig. 1, the superradiance leads to uniform spin polarization in $x$-direction and the Rydberg-mediated repulsion competes with this and tends to break the even-odd lattice translation symmetry.

Up the $V$-axis, at $g=0$ the magnetisations of the system can change discontinuously (at $V=\Delta / 4$ in two dimensions) from a fully polarised state $\left(\mathrm{FP}_{\uparrow}\right)$ to an antiferromagnetic excitation pattern (AFM). This AFM phase breaks a discrete even-odd translation symmetry

$$
T_{\text {lat }}:\left[\sigma_{e, o}^{\alpha}\right] \rightarrow\left[\sigma_{o, e}^{\alpha}\right]
$$

that can lead to different sublattice magnetisations as depicted in Fig. 2. Here, $T_{\text {lat }}$ exchanges the even (e) and odd (o) sublattice index of the atomic variables $\sigma^{\alpha}$ with $\alpha=\{x, y, z\}$ in the Hamiltonians in Eqs. (1-2). As described further in the caption of Fig. 2, the AFM+SR coexistence 
phase has a curious feature, namely that it is split in two regions, that are delimited by a touching point of two second-order phase transition lines of the $\mathrm{SR}_{\mathrm{UNI}}$ and the plain $\mathrm{AFM}$ phase at $V /|\Delta|=$ 1/2. For this special value, the effective magnetic field on one sublattice vanishes. At this point, however, the transition becomes discontinuous. Direct, continuous transitions between phases with different broken symmetries are rare and sometimes accompanied by deconfined quantum criticality, as in the ground state of frustrated quantum spin models, for example [49].

\section{Result 2: Even-odd sublattice peak in cavity spectrum}

A convenient feature of these quantum-optical quantum simulators is the ability to perform nondestructive measurements of the dynamics via cavity spectra. For the moving atomic quantum gas and the associated polaritonic density excitations, such measurements have lead to fruitful experiment-theory comparison [50-54].

Here we show that the translation-symmetry breaking induced by the nearest-neighbor Rydbergdressed interaction $V$ leads to a novel collective mode and peak in the spectrum. Fig. 9 shows the cavity spectrum upon increasing $g$ from the $(\mathrm{AFM}+\mathrm{SR})$ coexistence phase with broken $T_{\text {lat }}$ symmetry into the $\mathrm{SR}_{\mathrm{UNI}}$ phase where translation symmetry is restored. We observe that the conventional normal-mode polariton picture first seen by Rempe and Kimble [55] -which is, with modifications, also applicable to the Dicke model [56]- becomes insufficient to describe the photon dynamics. By contrast, the even-odd mode softens in a specific way: both the real part of the pole (gap) and the imaginary part (damping rate) vanish with coupling constant (from the AFM+SR phase toward line 2 in Fig. 2) with

$$
\operatorname{Re}[v] \propto \pm \sqrt{g_{c}-g}, \quad \operatorname{Im}[v] \propto-\left(g_{c}-g\right)
$$

where $g_{c}$ refers to the right boundary delimiting the AFM+SR phase. This is because the translational symmetry $T_{\text {lat }}$ affects the atomic sector only which does not couple directly to the photonic rate of dissipation $\kappa$. This is in contrast to Dicke-type models or superfluids out-of-equilibrium (see [57] and [58]) whose dynamics becomes purely overdamped/imaginary at the transition [56], that is, the real part of the mode vanishes first. 


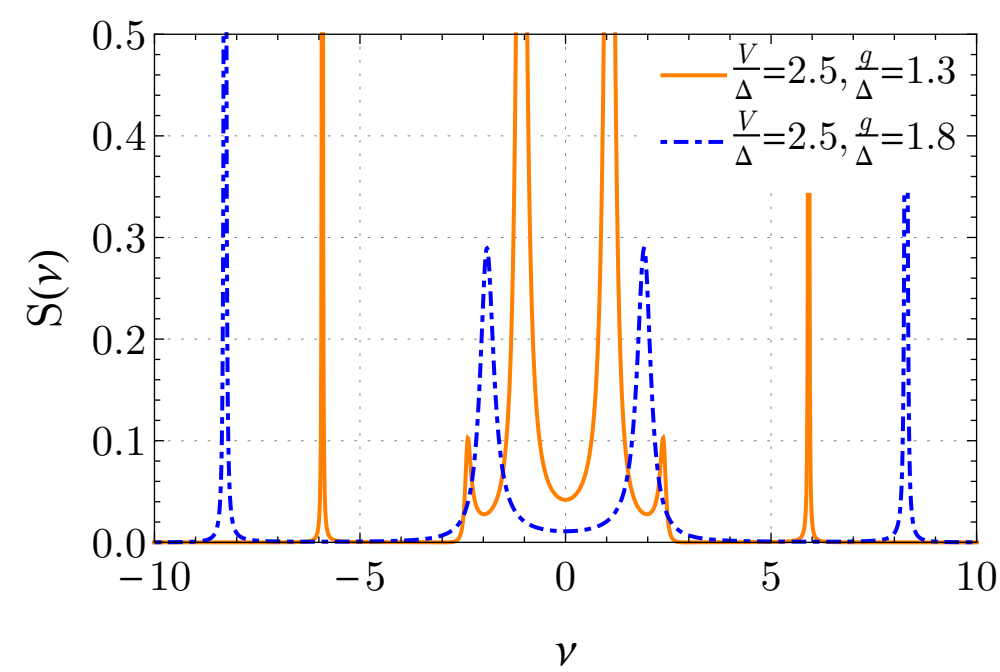

(a)

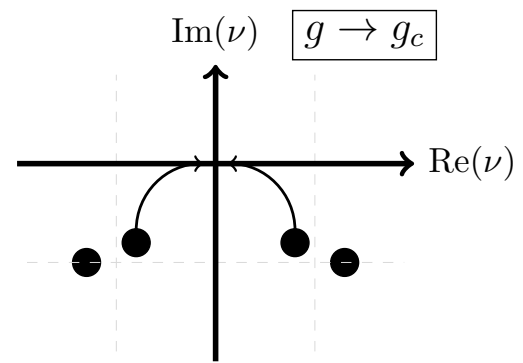

(b)

Figure 3. Even-odd sublattice peak in the cavity spectrum (peak close to zero frequency of the orange, solid line), which appears when the translational lattice symmetry $T_{\text {lat }}$ is spontaneously broken by the Rydbergdressed nearest-neighbor interaction $V$. The two cavity spectra are computed for the positions labeled by (x) in Fig. 2. The blue, dashed line has the two polariton peaks in the uniform $\mathrm{SR}_{\mathrm{UNI}}$ phase with the photonic branch around the cavity frequency $\omega_{0} / \Delta=2.0$. The orange, solid line is the spectrum in the $\mathrm{AFM}+\mathrm{SR}$ regime with broken $T_{\text {lat }}$; it shows the prominent even-odd peak, which becomes soft toward the phase boundary (the right edge of the yellow strip in Fig. 2. (b) Low-energy pole structure of the even-odd sublattice peak, where both the real and the imaginary part of the poles vanish simultaneously as $g \rightarrow g_{c}$ according to Eq. (7).

\section{Result 3: Photon number oscillations}

We now account for a non-zero rate of atomic spontaneous emission $\gamma \neq 0$. Specific details of a given quantum-optical implementation (see Sec. II) will determine which set of Lindblad operators and additional atomic levels need to be accounted for. In order to gain a first qualitative picture, we model an effective decay rate with $\mathcal{L}_{\gamma}[\rho]$ in Eq. (3) between the effective spin-up and spindown states $(|1\rangle$ and $|0\rangle$ in Fig. 7. We expect $\gamma$ to become larger once the detuning to the shorter

lived excited states is decreased; it is generally true that the effective ground state levels inherit a finite lifetime from admixing a short-lived state. For a specific experimental set-up, one may also include other types of atomic losses or dephasing.

This at first sight innocuous change has interesting consequences. Even qualitative features of 


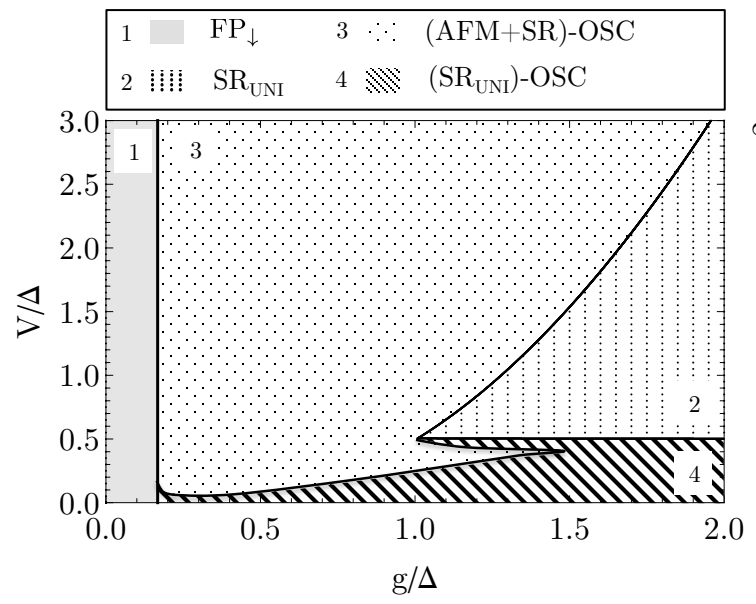

(a)

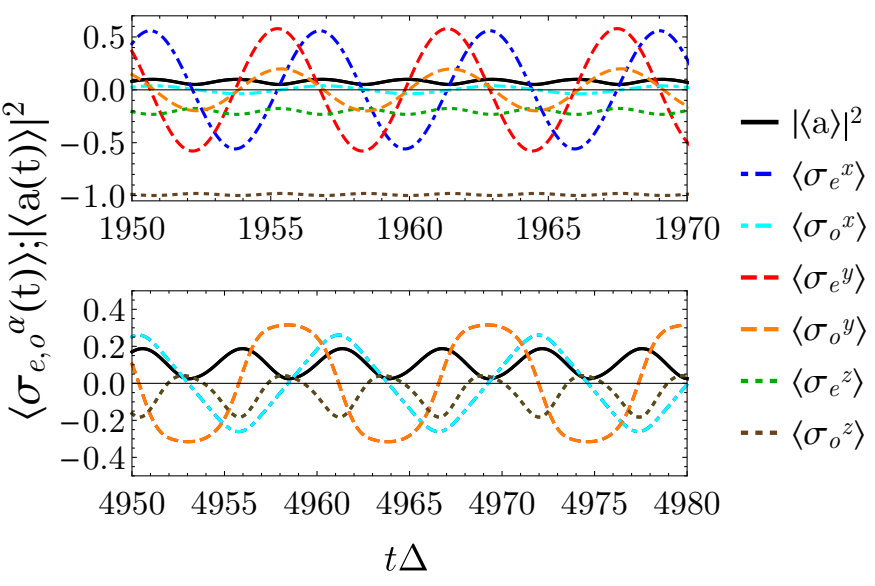

(b)

Figure 4. (a) Supplementing the phase diagram Fig. 2 in the $(\mathrm{V} / \Delta, \mathrm{g} / \Delta)$-plane by a small amount of atomic dissipation $\gamma / \Delta=0.01$ with $\left(\omega_{0} / \Delta=2.0, \kappa / \Delta=0.2\right)$ yields a different picture. In comparison to the $\gamma / \Delta=0.0$ case (compare Fig. 2), there are no stable steady-states with a broken lattice symmetry $T_{\text {lat }}$ any more. Instead, the system can show persistent oscillations in time. (b) Depiction of the non-uniform (top, $(\mathrm{AFM}+\mathrm{SR})-\mathrm{OSC}$ ) oscillations and the uniform (bottom, $\left.\mathrm{SR}_{\mathrm{UNI}}-\mathrm{OSC}\right)$ oscillations that characterize the long time limit behavior of Eqs. (12-15) with finite $\gamma$. Parameters for the upper plot are $(V / \Delta=g / \Delta=0.5)$, the lower plot is obtained at $(V / \Delta=0.3, g / \Delta=1.2)$.

Fig. 2 are drastically changed (although experimentally, for far enough detuned intermediate, excited states and rapid, enough ramps of the spin-light coupling $g$ these $\gamma$-induced changes may not be immediately visible). Allowing for a small $\gamma$, see Fig. 4, in particular wipes out the stable AFM phase and introduces a fully downward polarized state $\mathrm{FP}_{\downarrow}$ as well as a novel oscillatory coexistence phase $(\mathrm{AFM}+\mathrm{SR})$-OSC. Here also the photon field amplitude oscillates which can be detected by time-resolved measurements of the intensity of the light leaving the cavity.

At the root of these effect is $\mathcal{L}_{\gamma}[\rho]$ in Eq. (3): it explicitly breaks the discrete symmetry $G$ given by the product of time-reversal: $\mathcal{T}_{\ell}=-i \sigma_{\ell}^{y} K_{\ell}, t \rightarrow-t$ (for a spin $s=1 / 2$ ) and spin rotation around the y-axis by $\pi: D_{y, \pi}^{1 / 2, \ell}=-i \sigma_{\ell}^{y}$. Here $K_{\ell}$ is the complex conjugation operator such that $\mathcal{G}_{\ell}=D_{y, \pi}^{1 / 2, \ell} \mathcal{T}_{\ell}=-K_{\ell}$. If we write $G=\Pi_{\ell=1}^{N} \mathcal{G}_{\ell}$ we have $G H G^{-1}=H$. In particular, this implies for steady states $\left\langle\sigma_{e, o}^{y}\right\rangle=\left\langle G \sigma_{e, o}^{y} G^{-1}\right\rangle=-\left\langle\sigma_{e, o}^{y}\right\rangle \stackrel{!}{=} 0$ if $\gamma=0$. For $\gamma \neq 0$, the spins can start developing expectation values also in the $y$-direction. This offers new possibilities for the spin dynamics such as anomalous spin precession [28] not available in equilibrium.

We show the phases for a further range of parameters $(\Delta / \gamma, g / \gamma)$ space for a fixed strength $V$ of 


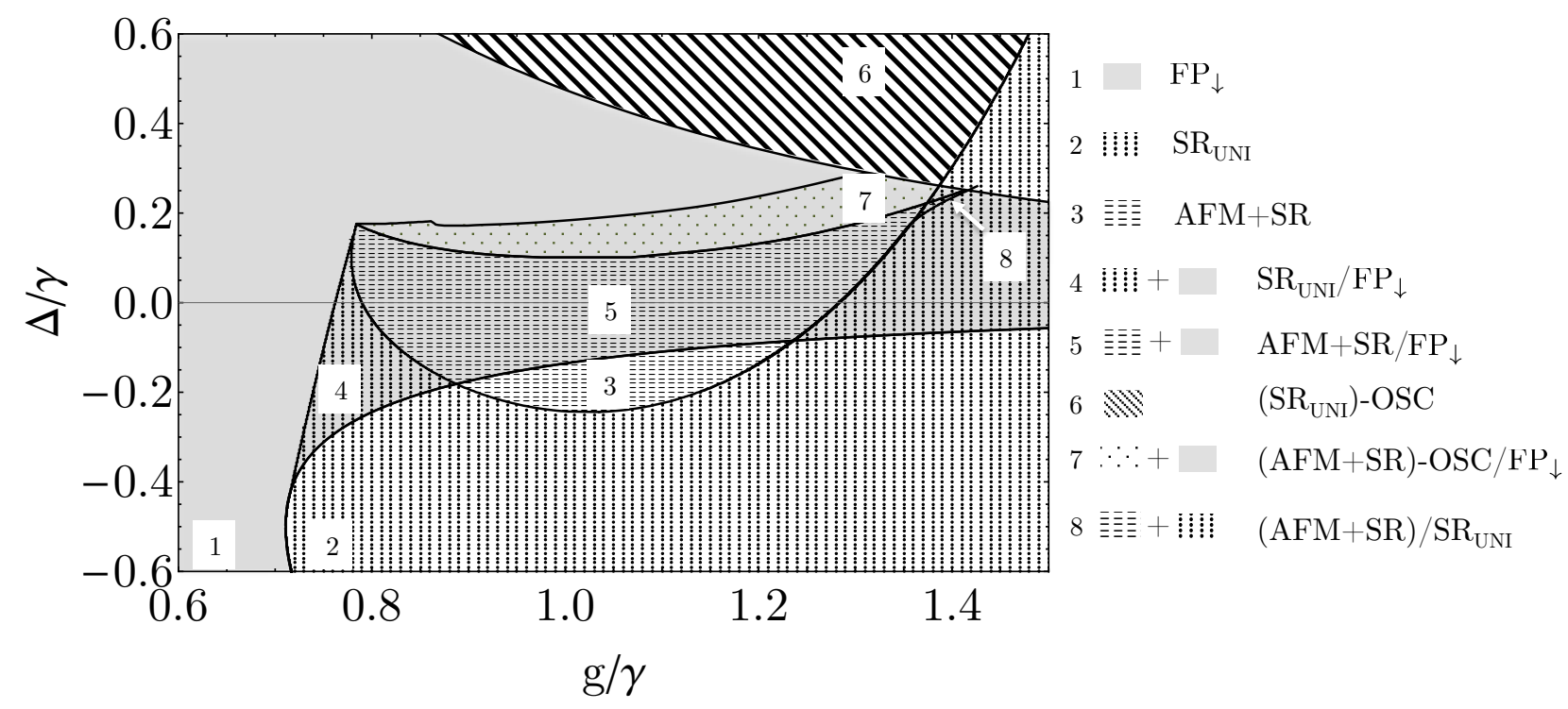

Figure 5. Non-equilibrium steady-state phase diagram of Eqs. (1-4) with finite, atomic spontaneous emission $\left(\kappa / \gamma=0.2, V / \gamma=1.8, \omega_{0} / \gamma=2.0\right)$. Apart from time-independent states, the dynamics also realises limit cycles where atomic and photon components show persistent oscillations in time. Oscillations can be uniform or different on the even/odd sublattice, see Fig. 6. Depending on the initial configuration, the system can reach different long-time fixpoints. Bistabilities occur whenever two phases overlap (see legend). Crystalline antiferromagentic order only occurs together with superradiance (AFM+SR).

the Rydberg interaction in two spatial dimensions in Fig. 5. In mean-field theory we distinguish five phases in the long time limit. Three are steady-states denoted as $\left(\mathrm{FP}_{\downarrow}, \mathrm{SR}_{\mathrm{UNI}}, \mathrm{AFM}+\mathrm{SR}\right.$, see also Tab. I) and two are stable limit cycles. The Lindblad operators try to drive the system into an empty state without any excitations; consequently AFM order can only occur in the presence of a coherent drive, i.e. in coexistence with a photon condensate $\langle a\rangle$. In the latter phases, the system exhibits oscillations in both atomic and photonic components, since the atomic dynamics couples back to the photon sector through Eq. (17). The oscillations can be uniform in all components $\left(\mathrm{SR}_{\mathrm{UNI}}\right)-\mathrm{OSC}$ or different on the sublattices $(\mathrm{AFM}+\mathrm{SR})-\mathrm{OSC}$.

One point of view to interpret the oscillations data in Fig. 6 is that the (collective) spin oscillator (see also Ref. 60) and the cavity oscillator synchronize with each other after a sufficient amount of time (see also Ref. 61). Additionally, the system can show bistabilities, meaning that the eventual fate of the system in the long-time limit depends on the initial conditions. However, we also find a small strip in the phase diagram where the system is bistable between AFM + SR and a $\mathrm{SR}_{\mathrm{UNI}}$ phase. We will describe the phases more in Sec IV C. 


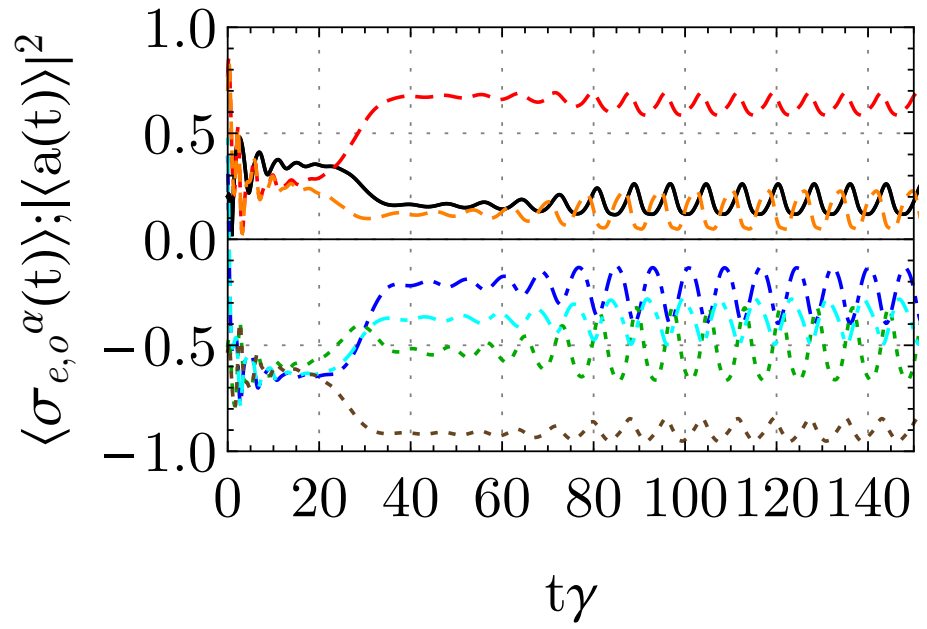

(a)

$$
\begin{aligned}
& -|\langle\mathrm{a}\rangle|^{2} \\
& =-\left\langle\sigma_{e^{x}}\right\rangle \\
& =-\left\langle\sigma_{o}{ }^{x}\right\rangle \\
& --\left\langle\sigma_{e^{y}}\right\rangle \\
& =-\left\langle\sigma_{o}{ }^{y}\right\rangle \\
& =-\left\langle\sigma_{e^{z}}\right\rangle \\
& =-\left\langle\sigma_{o}^{z}\right\rangle
\end{aligned}
$$

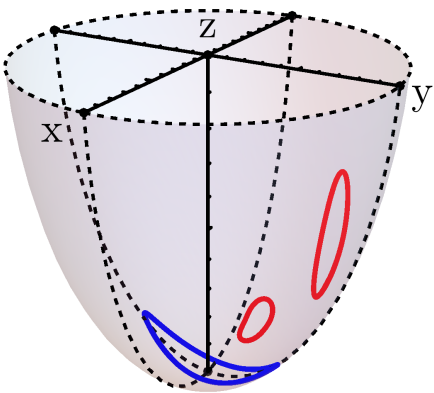

(b)

Figure 6. (a): Persistent spin and photon-field oscillations appear in the (AFM+SR)-OSC phase for $\Delta>0$ close to the $(\mathrm{AFM}+\mathrm{SR})$ stable region for the parameters used in Fig. ID for $g / \gamma=1.1, \Delta / \gamma=0.15$. Note that between $t \gamma \approx 10-25$ the amplitudes display (quasi-) plateaus followed by a rapid in/decrease toward their final mean values; and only then the oscillations begin and persist. For moving atomic gases in a cavity, we note that Ref. 59 found "pre-thermalized" plateaus in the time evoluation for the order parameter by solving Fokker-Planck type kinetic equations. (b): Illustration of the atomic components for the limit cycles on the lower part of the Bloch sphere. The two upper lineshapes (red) depict the lines traced by the oscillations on the even and the odd sublattice in the (AFM+SR)-OSC phase, as depicted in (a). The lower lineshape illustrates a limit cycle of uniform oscillations of the atomic components in the $\mathrm{SR}_{\mathrm{UNI}}$ phase.

\section{IDEAS FOR QUANTUM-OPTICAL IMPLEMENTATION OF THE MODEL}

We seek an implementation which realizes the Hamiltonian in Eqs. $(1,2)$. At the core of our model is the "two dipoles in-one" unit depicted in Fig. 7. As described in the caption, Dipole 1 could be created by weakly admixing a relatively low quantum number Rydberg level $(n \sim 30)$ to a set of long-lived hyperfine split states $|1\rangle$ and $|0\rangle$. Dipole 2 couples to the cavity via two far-detuned, excited states $|d\rangle,|e\rangle$.

The atomic levels we consider to realize an effective spin system could be the hyperfine-structure manifold of the ground states of ${ }^{87} \mathrm{Rb}$. Typically this is the $5^{2} S_{1 / 2}$ state split into the $F=1$ and the $F=2$ manifold such that $|0\rangle=|\downarrow\rangle=\left|F=1, m_{F}=-1\right\rangle$ and $|1\rangle=|\uparrow\rangle=\left|F=2, m_{F}=-2\right\rangle$. Here, cavity-assisted Raman transitions couple the $(|0\rangle,|1\rangle)$ ground-states via adiabatic elimination of the detuned excited states $(|d\rangle,|e\rangle)$ to the cavity [40,45]. Then, the cavity is (indirectly) pumped 


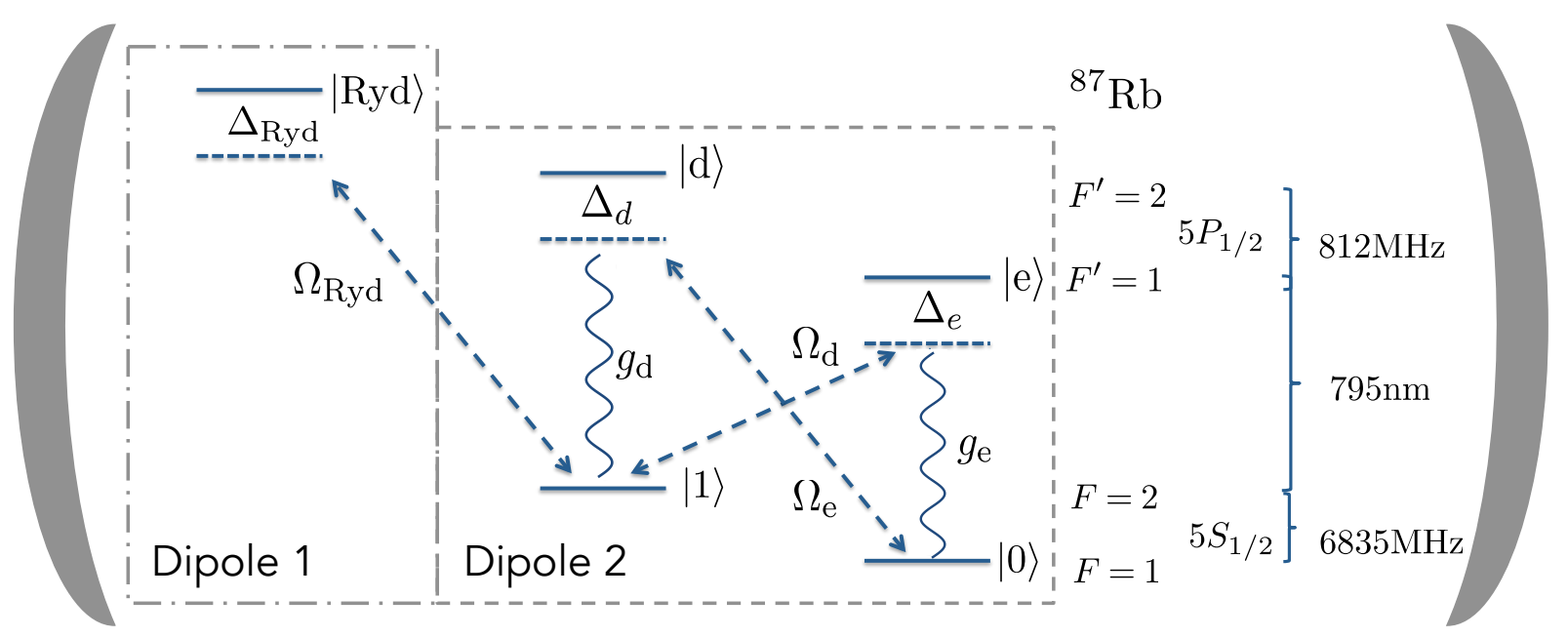

Figure 7. Blueprint for the wanted "two dipoles in-one" unit and the Hamiltonian Eqs. $(1,2)$, which allows two independent photonic force carriers to couple to the atomic spin. The effective spin degree of freedom is encoded in the levels $|1\rangle$ and $|0\rangle$. The resonator mirrors (grey shades) confine the optical photon mode, which couples to the effective spin via $g_{d}$ and $g_{e}$. Dipole 1: A dressing laser can weakly admix a Rydberg level to a ground-state $|1\rangle \rightarrow|1\rangle+\frac{\Omega_{\mathrm{Ryd}}}{2 \Delta_{\mathrm{Ryd}}}|\mathrm{Ryd}\rangle=|\tilde{1}\rangle$. We want the resulting effective potential $V_{\ell m}^{\text {eff }}$ between $|\tilde{1}\rangle_{\ell}$ and $|\tilde{1}\rangle_{m}$ states to be predominantly nearest-neighbor in a square lattice spaced by an optical wavelength. Complex potentials including angle-dependencies can be realized [5, 25, 26]. Dipole 2: The double Raman scheme [40] provides a tunable coupling $\sim\left(a+a^{\dagger}\right) \sigma_{\ell}^{x}$ to the optical cavity. Choosing the lattice and cavity modefunction as in Fig. 1 results in a homogeneous coupling $g$, that is, all the spins couple in the same way to the cavity. This provides the infinite-range coupling between all the spins. The depicted level scheme corresponds to the ground state manifold $5 S_{1 / 2}$ and the first excited state manifold $5 P_{1 / 2}$ of ${ }^{87} \mathrm{Rb}$ including their typical frequency splittings.

with photons from the transversal pumping-laser that scatter off the atoms and populate the cavity mode. In that way, the pump is "hidden" in the atom-photon coupling $g$ : it is the counter-rotating terms that stabilize non-trivial steady-states with finite excitation number.

The second part of the pumping scheme, denoted as Dipole 1 in Fig. 7, consists of admixing a small part of a Rydberg state to the state $|1\rangle$ that is also coupled to the cavity. To first-order in perturbation theory of the driving, the ground-state becomes dressed with a small fraction of the excited state $|\tilde{1}\rangle \approx|1\rangle+\frac{\Omega_{\mathrm{Ryd}}}{2 \Delta_{\mathrm{Ryd}}}|\mathrm{Ryd}\rangle+O\left(\frac{\Omega_{\mathrm{Ryd}}}{2 \Delta_{\mathrm{Ryd}}}\right)^{2}$, where $\Omega_{\mathrm{Ryd}}$ is the Rabi-frequency and $\Delta_{\mathrm{Ryd}}$ is the detuning from the Rydberg level. Ground-states $\left\{|\tilde{1}\rangle_{i},|\tilde{1}\rangle_{j}\right\}$ interact then with a dressed Rydberg interaction that can be controlled by changing $\left(\Omega_{\mathrm{Ryd}}, \Delta_{\mathrm{Ryd}}\right)$ of the dressing laser [62-65]. Dressing 
schemes for Rydberg atoms on optical lattices have recently been experimentally realised [5] and the effective Rydberg potential depends strongly on the chosen Rydberg states [25]. An additional degree of freedom that allows for engineering anisotropic effective potentials with an angular dependence $V_{i j}^{\text {eff }}\left(r_{i j}, \theta_{i j}\right)$ can be introduced by employing states with angular momentum such as states from $P$-manifolds [25].

In a suitably chosen rotating frame of reference, derived in Appendix B, the parameters appearing in the Hamiltonian Eqs. $(1,2)$, can be expressed as follows. For the spin longitudinal field $\Delta$ and the effective cavity frequency $\omega_{0}$, we have

$$
\Delta=-\Delta_{1}+\frac{\Omega_{\mathrm{Ryd}}^{2}}{4 \Delta_{\mathrm{Ryd}}}, \quad \omega_{0}=N \frac{g_{e}^{2}}{\Delta_{e}}+\omega_{a},
$$

with $\omega_{a}$ and $\Delta_{1}$ defined in Eq. (B13). The coherent coupling to the cavity can be tuned by the two external lasers $\Omega_{d, e}$ :

$$
g=\sqrt{N} \frac{g_{d, e} \Omega_{d, e}}{2 \Delta_{d, e}}, \quad \frac{\Omega_{d}}{4 \Delta_{d}}=\frac{\Omega_{e}}{4 \Delta_{e}}, \quad \frac{g_{d}^{2}}{\Delta_{d}}=\frac{g_{e}^{2}}{\Delta_{e}} .
$$

Finally, the Rydberg-mediated potential takes the general form [64]

$$
V_{\ell m}^{\mathrm{eff}}=\left(\frac{\Omega_{\mathrm{Ryd}}}{2 \Delta_{\mathrm{Ryd}}}\right)^{4} \frac{C_{6}}{r_{\ell m}^{6}+R_{c}^{6}},
$$

and the $V$ appearing in Eq. (1) evaluates Eq. (10) at a fixed $r_{\ell m}$ equal to the distance between neighboring lattice sites. Effectively step-like potentials are also possible. $R_{c}$ is the critical radius defined by $2 \Delta_{\mathrm{Ryd}} \equiv V\left(R_{c}\right)$ which yields $R_{c}=\left(\frac{C_{6}}{2 \hbar\left|\Delta_{\mathrm{Ryd}}\right|}\right)^{1 / 6}$. At smaller distances $r_{\ell m}<R_{c}$, dressing to doubly excited states becomes ineffective because of the large detuning $\left|\Delta_{\text {Ryd }}\right|+V_{\ell m}$. The soft-core potentials contain a number of additional resonances at $r_{i j} \ll R_{c}$, which are undesirable to realise clean interactions. To ensure the interacting atoms in an optical lattice interact via the clean van-der-Waals tail, it is more advantageous to address relatively low-lying Rydberg states with principal quantum numbers $n \sim 30$, as $R_{c}$ can then also shrink down to optical wavelengths. Additionally, we comment that complementary to optical lattices, two-dimensional arrays of microtraps have already been used [66] to trap single ${ }^{87} \mathrm{Rb}$ atoms with lattice spacings $\sim \mu m$. This would allow to use more highly excited Rydberg states for the dressing interaction bringing with it the advantage of longer lifetimes of higher lying Rydberg states. 


\section{COUPLED EVEN-ODD SUBLATTICE MEAN-FIELD MASTER EQUATIONS FOR ATOMS AND PHOTONS}

We now derive and solve the coupled mean-field master equations equations for both, the spin degrees of freedom and the photons for an infinite number of atomic spins $N \rightarrow \infty$. In absence of the short-range, nearest-neighbor interaction $V$, a mean-field ansatz actually represents the exact solution in the long time limit $t \rightarrow \infty$ [46]. We account for different spin expectation values on the even versus odd sublattice of the bipartite square lattice of Fig. 1. The goal is to allow for steady-states with spontaneously broken translational (even-odd interchange) symmetry.

To this end, we now approximate the solution of the full master equation

$$
\partial_{t} \rho=-i[H, \rho]+\mathcal{L}_{\kappa}[\rho]+\mathcal{L}_{\gamma}[\rho]
$$

by factorizing the spin part of density operator for all even sites as $\rho_{e}=\bigotimes_{\ell=1}^{N / 2} \rho_{e, \ell}$ and analogously for the odd sites $\rho_{o}=\bigotimes_{\ell=1}^{N / 2} \rho_{o, \ell}$. In the conclusions, Sec. V, we comment further on the prospects of capturing finite spatial correlations and fluctuations beyond mean-field. We further define the spin expectation values on the even and odd sublattices, respectively: $\left\langle\sigma_{e / o}^{\alpha}\right\rangle=\operatorname{Tr}\left[\rho_{e / o} \sigma_{e / o}^{\alpha}\right]$ where $\alpha$ refers to $(x, y, z)$. These six real-valued spin projections are complemented by two variables for the photon fields, $\langle a\rangle,\left\langle a^{\dagger}\right\rangle$, making it a total of eight variables to keep track of. This way, $\rho$ also includes entries to discriminate between the vacuum and a coherent photon field. The first four equations read

$$
\begin{aligned}
\partial_{t}\left\langle\sigma_{e}^{x}(t)\right\rangle & =\left\langle\sigma_{e}^{y}(t)\right\rangle\left[\Delta-2 V\left(\left\langle\sigma_{o}^{z}(t)\right\rangle+1\right)\right]-\frac{\gamma}{2}\left\langle\sigma_{e}^{x}(t)\right\rangle \\
\partial_{t}\left\langle\sigma_{e}^{y}(t)\right\rangle & =\left\langle\sigma_{e}^{x}(t)\right\rangle\left[2 V\left(\left\langle\sigma_{o}^{z}(t)\right\rangle+1\right)-\Delta\right]-2 g\left[\langle a(t)\rangle+\left\langle a^{\dagger}(t)\right\rangle\right]\left\langle\sigma_{e}^{z}(t)\right\rangle-\frac{\gamma}{2}\left\langle\sigma_{e}^{y}(t)\right\rangle \\
\partial_{t}\left\langle\sigma_{e}^{z}(t)\right\rangle & =2 g\left[\langle a(t)\rangle+\left\langle a^{\dagger}(t)\right\rangle\right]\left\langle\sigma_{e}^{y}(t)\right\rangle-\gamma\left(1+\left\langle\sigma_{e}^{z}(t)\right\rangle\right) \\
\partial_{t}\langle a(t)\rangle & =-\left(\kappa+i \omega_{0}\right)\langle a(t)\rangle-\frac{1}{2} i g\left(\left\langle\sigma_{e}^{x}(t)\right\rangle+\left\langle\sigma_{o}^{x}(t)\right\rangle\right)
\end{aligned}
$$

The equations for the odd sublattice spin projections follow from Eqs. (12-14) by exchanging the sublattice index $e \leftrightarrow o$. The complex conjugate of Eq. (15) completes the set of eight coupled equations. Here, we rescaled the photonic variable with $a(t) \rightarrow \sqrt{N}\langle a(t)\rangle$ a steady-state is macroscopically occupied in the thermodynamic limit and one may also define $\left\langle\sigma_{e, o}^{\alpha}\right\rangle \equiv \frac{1}{(N / 2)} \sum_{\ell \in \text { even,odd }}^{N} \sigma_{\ell}^{\alpha}$. 


\section{DERIVATION AND DETAILED DISCUSSION OF RESULTS}

\section{A. Result 1: Coexistence of superradiance and magnetic translation symmetry-breaking}

\section{Phase boundaries and order parameters with photon losses $(\kappa \neq 0, \gamma=0)$}

We first consider the case where the atoms do not decay spontaneously and the only loss-process is given by the Lindblad $\mathcal{L}_{\kappa}$, see Eq. (4). The Eqs. (12-15) with $\gamma=0$ conserve in this case a pseudo-angular momentum

$$
\left\langle\sigma_{e, o}^{x}\right\rangle^{2}+\left\langle\sigma_{e, o}^{z}\right\rangle^{2}=1
$$

provided we start from a low-entropy initial state for the spins (as discussed above in Subsec. IB), which fulfill this condition. Here $(e, o)$ refers to the even and odd sub lattice respectively. Due to the presence of time-reversal symmetry in the atomic channel, the steady-state of the system constrains $\left\langle\sigma_{e, o}^{y}\right\rangle=0$ (see the discussion above in Sec. ID). Fig. 2 is calculated, with photon losses, by numerically solving for the stationary states of Eqs. (12-15). We determine stability by inspecting the real parts of the eigenvalues from the corresponding stability matrix that is obtained by linearising Eqs. (12-15) to first oder in fluctuations around the steady-states.

Together with the constraint Eq. (16), the set of Eqs. (12-15) can be solved analytically. The homogeneous $\left(\left\langle\sigma^{x, z}\right\rangle \equiv\left\langle\sigma_{e}^{x, z}\right\rangle=\left\langle\sigma_{o}^{x, z}\right\rangle\right)$ steady-state solutions, which describe the Dicke superradiance transition are

$$
\begin{aligned}
\langle a\rangle & =\mp \frac{g\left\langle\sigma^{x}\right\rangle}{\omega_{0}-i \kappa}, \\
\left\langle\sigma^{x}\right\rangle & =\mp \frac{\sqrt{J-J_{c}} \sqrt{4 J+\Delta}}{\sqrt{2 J+V}}, \\
\left\langle\sigma^{z}\right\rangle & =\frac{J_{c}+\frac{\Delta}{4}}{2 J-J_{c}+\frac{\Delta}{4}} .
\end{aligned}
$$

We have defined $J=\frac{g^{2} \omega_{0}}{\kappa^{2}+\omega_{0}^{2}}$ and $J_{c}=\frac{\Delta}{4}-V$. A plot of the Eqs. (17)-(19) is illustrated in Fig. 8b. Starting in the antiferromagnetic phase and increasing the atom-light coupling $g$, a coexistence regime of superradiance $(\langle a\rangle \neq 0)$ and a phase with different sub-lattice magnetisations is predicted $\left(\left\langle\sigma_{e}^{z}\right\rangle-\left\langle\sigma_{o}^{z}\right\rangle \neq 0\right)$ which, due to Eq. (16) implies $\left\langle\sigma_{e}^{x}\right\rangle-\left\langle\sigma_{o}^{x}\right\rangle \neq 0$. Due to the finite longitudinal field $\Delta$, one sublattice is easier flipped than the other and the system realizes a "canted" antiferromagnet. If the atom-light coupling is increased even further, translational symmetry is restored and the 


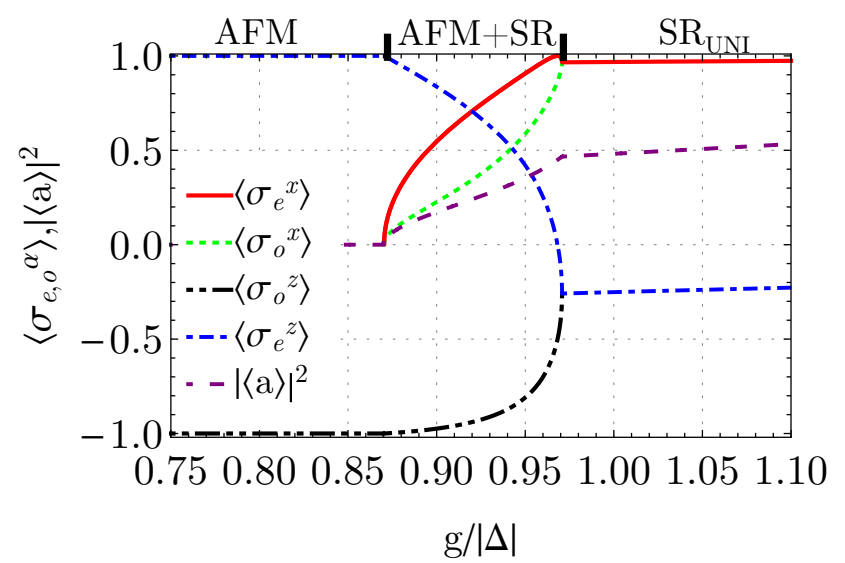

(a)

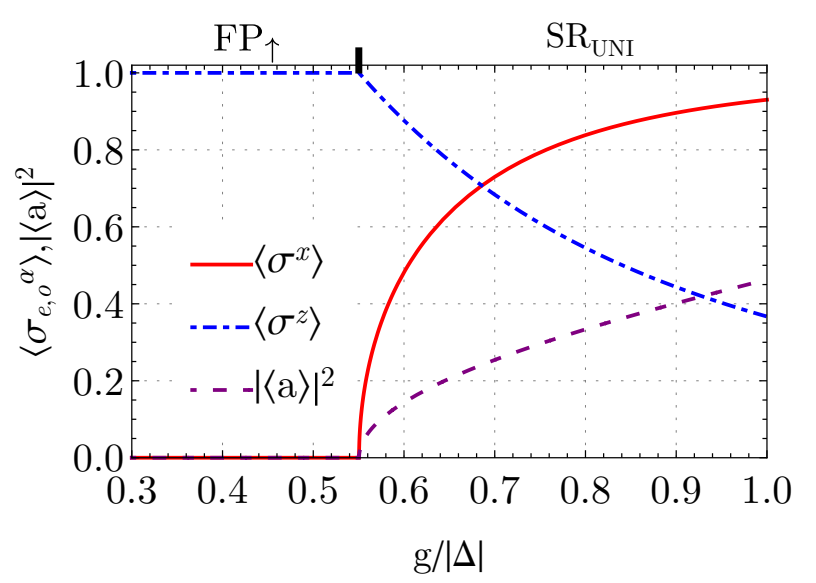

(b)

Figure 8. Steady-states for Eqs. (12-15) with $\gamma=0$ for the parameters $\omega_{0}=2.0|\Delta|, \kappa=0.2|\Delta|$ for the phases depicted in Fig. 2. (a) Behavior of the magnetisations and the coherent photon condensate in the plain antiferromagnet (AFM), in the coexistence regime $(\mathrm{AFM}+\mathrm{SR})$ and in the Dicke-phase $\left(\mathrm{SR}_{\mathrm{UNI}}\right)$ as the atom-light coupling $g$ is increased at a fixed value of $V=|\Delta|$. All transitions are continuous in the orderparameters. The plot in (b) shows the onset of superradiance as the atom-light coupling is increased, for a fixed value of $V=0.1|\Delta|$.

system realises a Dicke superradiant phase. This can be seen by tracking the evolution of the magnetisation as $g$ is increased in Fig. 8a.

We now derive analytical expressions for the phase-transition lines. First, we transform the meanfield equations (12-15) in frequency space via Fourier transformation

$$
O(t)=\frac{1}{\sqrt{2 \pi}} \int_{-\infty}^{\infty} e^{-i v t} O(v) d v, \quad O^{\dagger}(t)=\frac{1}{\sqrt{2 \pi}} \int_{-\infty}^{\infty} e^{-i v t} O^{\dagger}(-v) d v
$$

In general, one should add Markovian quantum noise-operators with zero-mean to the photonic and atomic set of the mean-field master equations that result from the interaction of the atomcavity system with the vacuum modes outside of the cavity. These we denote $\mathcal{F}_{e, o}^{\alpha}(v)$ as the atomic and $\mathcal{F}^{a}(v)$ as the photonic noise-operators in frequency space.

Next, we add back fluctuations to Eqs. (12-15)

$$
\begin{gathered}
\left\langle\sigma_{e, o}^{\alpha}(v)\right\rangle \rightarrow\left\langle\sigma_{e, o}^{\alpha}\right\rangle \delta(v) \sqrt{2 \pi}+\delta \sigma_{e, o}^{\alpha}(v) \\
\sqrt{N}\langle a(v)\rangle \rightarrow \sqrt{N}\langle a\rangle \delta(v) \sqrt{2 \pi}+\delta a(v),
\end{gathered}
$$


where the steady-states are denoted as $\left\langle\sigma_{e, o}^{\alpha}\right\rangle$ with $\alpha=(x, y, z)$ and $\langle a\rangle$ is the expectation value for a coherent photon condensate. Here, $\delta \sigma_{e, o}^{\alpha}(v)$ and $\delta a(v)$ describe quantum fluctuations about the semi-classical steady-state and $\delta(v)$ denotes a delta function in frequency space. At long times, we may neglect second-order terms in the fluctuations by assuming that the steady-state values are large compared to the associated fluctuations in the thermodynamic limit $N \rightarrow \infty$.

The now linearized equations can be cast in matrix form.

$$
\mathcal{F}(v)=\delta(v) f(\sigma)+\boldsymbol{G}_{R}^{-1}(v) \cdot \boldsymbol{\delta} \boldsymbol{\sigma}(v)
$$

where the fluctuations around the steady state are collected in $\delta \sigma(v)$ and the noise-operators are collected into $\mathcal{F}(v)$ :

$$
\begin{aligned}
\delta \sigma^{T}(v) & =\left(\delta \sigma_{e}^{x}(v), \delta \sigma_{e}^{y}(v), \delta \sigma_{e}^{z}(v), \delta a(v), \delta a^{\dagger}(-v), \delta \sigma_{o}^{x}(v), \delta \sigma_{o}^{y}(v), \delta \sigma_{o}^{z}(v)\right) \\
\mathcal{F}^{T}(v) & =\left(\mathcal{F}_{e}^{x}(v), \mathcal{F}_{e}^{y}(v), \mathcal{F}_{e}^{z}(v), \mathcal{F}^{a}(v), \mathcal{F}^{a^{\dagger}}(-v), \mathcal{F}_{o}^{x}(v), \mathcal{F}_{o}^{y}(v), \mathcal{F}_{o}^{z}(v)\right)
\end{aligned}
$$

The function $f(\sigma)$ is associated with the coherent part of the steady-states and thus only leads to a zero-frequency peak in the cavity-spectrum. The responses of the fluctuations $\delta \sigma$ to the noise or 'driving-forces' $\mathcal{F}$ is encoded by the retarded Green-function $\boldsymbol{G}_{R}(v)$, its inverse $\boldsymbol{G}_{R}^{-1}(v)$ is given as:

$$
\left(\begin{array}{cccccccc}
\frac{1}{2}(\gamma-2 i v) & 2 V\left(\left\langle\sigma_{o}^{z}\right\rangle+1\right)-\Delta & 0 & 0 & 0 & 0 & 0 & 2 V\left\langle\sigma_{e}^{y}\right\rangle \\
\Delta-2 V\left(\left\langle\sigma_{o}^{z}\right\rangle+1\right) & \frac{1}{2}(\gamma-2 i v) & 2 g\left(\langle a\rangle+\left\langle a^{\dagger}\right\rangle\right) & 2 g\left\langle\sigma_{e}^{z}\right\rangle & 2 g\left\langle\sigma_{e}^{z}\right\rangle & 0 & 0 & -2 V\left\langle\sigma_{e}^{x}\right\rangle \\
0 & -2 g\left(\langle a\rangle+\left\langle a^{\dagger}\right\rangle\right) & \gamma-i v & -2 g\left\langle\sigma_{e}^{y}\right\rangle & -2 g\left\langle\sigma_{e}^{y}\right\rangle & 0 & 0 & 0 \\
\frac{1}{2} i g & 0 & 0 & \kappa-i\left(v-\omega_{0}\right) & 0 & \frac{1}{2} i g & 0 & 0 \\
-\frac{1}{2} i g & 0 & 0 & 0 & \kappa-i\left(v+\omega_{0}\right) & -\frac{1}{2} i g & 0 & 0 \\
0 & 0 & 2 V\left\langle\sigma_{o}^{y}\right\rangle & 0 & 0 & \frac{1}{2}(\gamma-2 i v) & 2 V\left(\left\langle\sigma_{e}^{z}\right\rangle+1\right)-\Delta & 0 \\
0 & 0 & -2 V\left\langle\sigma_{o}^{x}\right\rangle & 2 g\left\langle\sigma_{o}^{z}\right\rangle & 2 g\left\langle\sigma_{o}^{z}\right\rangle & \Delta-2 V\left(\left\langle\sigma_{e}^{z}\right\rangle+1\right) & \frac{1}{2}(\gamma-2 i v) & 2 g\left(\langle a\rangle+\left\langle a^{\dagger}\right\rangle\right) \\
0 & 0 & 0 & -2 g\left\langle\sigma_{o}^{y}\right\rangle & -2 g\left\langle\sigma_{o}^{y}\right\rangle & 0 & -2 g\left(\langle a\rangle+\left\langle a^{\dagger}\right\rangle\right) & \gamma-i v
\end{array}\right)
$$

In the case of second order transitions, the order-parameters $\left(\langle a\rangle,\left\langle\sigma_{e, o}^{x}\right\rangle,\left\langle\sigma_{e, o}^{z}\right\rangle\right)$ change continuously at the phase transitions. We obtain analytical expressions for the phase boundaries by solving

$$
\lim _{v \rightarrow 0} \operatorname{Det}\left[\boldsymbol{G}_{\mathrm{R}}^{-1}(v)\right]=\alpha^{2}=0 .
$$

The zeroth-order frequency component refers to a possible gap $\alpha^{2}$ of the system that will close continuously ( $\lim _{g \rightarrow g_{c}} \alpha^{2} \rightarrow 0$ ) when the phase transition is approached by increasing the atomlight coupling $g$. We arrive at the set of transition lines given by Eq. (29), Eq. (30) and Eq. (32) that are depicted as black lines in Fig. 2 where they match the numerically calculated transitions. 
The open character of the system becomes manifest in the expressions for the phase boundaries as all transitions explicitly depend on the rate of photonic dissipation $\kappa$. Starting from the $\mathrm{FP}_{\uparrow}$ phase, the Dicke superradiance transition in presence of the Rydberg interaction sets in at the critical coupling strength:

$$
\begin{aligned}
& g_{\mathrm{c}, 1}=\frac{\sqrt{\kappa^{2}+\omega_{0}^{2}} \sqrt{\omega_{0}(\Delta-4 V)}}{2 \omega_{0}}, \\
& V_{\mathrm{c}, 1}=\frac{\Delta}{4}-\frac{g^{2} \omega_{0}}{\kappa^{2}+\omega_{0}^{2}} .
\end{aligned}
$$

The finite Rydberg-dressed interaction $V$ modifies the effective longitudinal field experienced by the spins which shifts the position of the superradiant condensate in comparison to the $V=0$ case. Eq. (28) collapses to the familiar Dicke superradiance transition in the case $V \rightarrow 0$ [40]. The crossover from the coexistence regime to the Dicke superradiant phase is given by:

$$
\begin{aligned}
& V_{\mathrm{c}, 2}= \\
& {\left[4 g^{2} \omega_{0}\left(4 g^{2} \omega_{0}+\sqrt{\Delta^{2}\left(\kappa^{2}+\omega_{0}^{2}\right)^{2}-8 \Delta g^{2} \omega_{0}\left(\kappa^{2}+\omega_{0}^{2}\right)+80 g^{4} \omega_{0}^{2}}\right)\right.} \\
& +\Delta\left(\kappa^{2}+\omega_{0}^{2}\right) \sqrt{\Delta^{2}\left(\kappa^{2}+\omega_{0}^{2}\right)^{2}-8 \Delta g^{2} \omega_{0}\left(\kappa^{2}+\omega_{0}^{2}\right)+80 g^{4} \omega_{0}^{2}} \\
& \left.+\Delta^{2}\left(\kappa^{2}+\omega_{0}^{2}\right)^{2}\right] / 8\left(\kappa^{2}+\omega_{0}^{2}\right)\left(\Delta\left(\kappa^{2}+\omega_{0}^{2}\right)+2 g^{2} \omega_{0}\right) .
\end{aligned}
$$

The transition line from the AFM phase to the coexistence AFM+SR regime is given by $(-\Delta<0)$

$$
\begin{aligned}
g_{\mathrm{c}, 3} & =\frac{\sqrt{\Delta} \sqrt{\kappa^{2}+\omega_{0}^{2}} \sqrt{4 V-\Delta}}{2 \sqrt{2} \sqrt{V} \sqrt{\omega_{0}}}, \\
V_{\mathrm{c}, 3} & =\frac{\Delta^{2}\left(\kappa^{2}+\omega_{0}^{2}\right)}{4\left(\Delta\left(\kappa^{2}+\omega_{0}^{2}\right)-2 g^{2} \omega_{0}\right)} .
\end{aligned}
$$

We note that the coexistence line diverges $V_{\mathrm{c}, 3} \rightarrow \infty$ as $\lim _{g \rightarrow g_{\star}}$ with $g_{\star}=\left(\sqrt{\Delta} \sqrt{\kappa^{2}+\omega_{0}^{2}}\right) /\left(\sqrt{2} \sqrt{\omega_{0}}\right)$ Moreover, on a mean-field level, there is a touching point $g_{t}$ of two second-order phase transition lines that can be found by equating Eq. (30) and Eq. (32) which yields $g_{t}=\left(\sqrt{\Delta} \sqrt{\kappa^{2}+\omega_{o}^{2}}\right)\left(2 \sqrt{\omega_{o}}\right)$ and $V_{c, 3}\left(g_{t}\right)=\Delta / 2$ marks the point where the effective longitudinal field on one of the sublattices vanishes. On a mean-field level, we find a multi-critical point, where all second-order phase transition lines meet on the $g=0$-axis at $V=\Delta / 4$.

In Appendix A, we analyze a $T=0$ equilibrium spin model with the same phases as Fig. 2 upon identifying one spin interaction constant with cavity parameters. Dynamics and statistics remains drastically different in the non-equilibrium case, however. 


\section{B. Result 2: Even-odd sublattice peak in cavity spectrum}

1. Derivation of the cavity output spectrum $(\kappa \neq 0, \gamma=0)$

Here we calculate the frequency-resolved cavity output spectrum for the light that leaks from the imperfect cavity mirrors within a standard input-output theory [40, 67, 68]. We find that every phase in Fig. 2 shows a characteristic cavity output spectrum making it possible to experimentally distinguish one phase from the other. The input fields are related to the output fields by the relation

$$
\begin{gathered}
a_{\text {out }}(v)=\sqrt{2 \kappa} a(v)-a_{\text {in }}(v), \\
a_{\text {out }}^{\dagger}(-v)=\sqrt{2 \kappa} a^{\dagger}(-v)-a_{\text {in }}^{\dagger}(-v) .
\end{gathered}
$$

The annihilation operators $\left(a_{\text {out }}(v), a_{\text {in }}(v), a(v)\right)$ correspond to the output field, the vacuum input field, and the intra cavity field, respectively and we have used $\mathcal{F}_{a}(v)=\sqrt{2 \kappa} a_{\text {in }}(v)$. The Markovian quantum noise operators with zero mean are determined by their second-order correlation functions. For the photonic channel they read

$$
\left\langle a_{\text {in }}\left(v^{\prime}\right) a_{\text {in }}^{\dagger}(-v)\right\rangle=\delta\left(v+v^{\prime}\right)
$$

We solve Eq. (23) for $a_{\text {out }}(v)$ and $a_{\text {out }}^{\dagger}(-v)$ together with Eq. (35) to obtain the output spectrum for a vacuum input field

$$
S(v)=\left\langle a_{\mathrm{out}}^{\dagger}(v) a_{\mathrm{out}}(v)\right\rangle=2 \kappa\left\langle\delta a^{\dagger}(v) \delta a(v)\right\rangle=2 \kappa \int_{-\infty}^{\infty} e^{-i v \tau}\left\langle\delta a^{\dagger}(0) \delta a(\tau)\right\rangle d \tau .
$$

The unnormalized fluorescence spectrum $S(v)$ is proportional to finding a photon at frequency $v$ and thus displays the position and the spectral weight of the resonance energies of hybridized atom-cavity modes. We only depict cavity-spectra in the $\gamma=0$ limit. We have investigated the effect of spontaneous emission on the cavity spectra in the $V \rightarrow 0$ limit previously [46] and found that it can induce a frequency asymmetry in the cavity spectrum since atomic excitations can leave the cavity directly by emission into free space. The cavity spectra for $\kappa \neq 0, \gamma=0$ can be obtained for every phase in Fig. 2. In the fully polarized phase $\left(\langle a\rangle=\left\langle\sigma^{x}\right\rangle=0,\left\langle\sigma^{z}\right\rangle=1\right)$ for $g\left\langle g_{c, 1}\right.$ and $-\Delta<0$, the cavity spectrum is obtained as

$$
\begin{aligned}
S_{0}(v) & =\frac{16 \kappa^{2} g^{4}(\Delta-4 V)^{2}}{\Omega_{0} \Omega_{0}^{*}}, \\
\Omega_{0} & =\left((\kappa-i v)^{2}(-\Delta+v+4 V)(\Delta+v-4 V)+4 g^{2} \omega_{0}(\Delta-4 V)+\omega_{0}^{2}(-\Delta+v+4 V)(\Delta+v-4 V)\right)
\end{aligned}
$$


and is depicted in Fig. 9c. In the limit $V \rightarrow 0$ it reduces to the familiar expression obtained in Ref. [40]. In the AFM phase $\left(\left\langle\sigma_{e}^{z}\right\rangle=-1,\left\langle\sigma_{o}^{z}\right\rangle=1,\langle a\rangle=\left\langle\sigma^{x}\right\rangle=0\right)$, the spectrum is given by

$$
\begin{aligned}
S_{3}(v)= & \frac{64 \kappa^{2} g^{4} V^{2}\left(\Delta^{2}-4 \Delta V+\omega^{2}\right)^{2}}{\Omega_{2} \Omega_{2}^{*}} \\
\Omega_{3}= & 8 g^{2} V \omega_{0}\left(\Delta^{2}-4 \Delta V+\omega^{2}\right)+(\omega-\Delta)(\Delta+\omega)(\kappa-i \omega)^{2}(-\Delta+4 V+\omega)(\Delta-4 V+\omega) \\
& +\omega_{0}^{2}(\omega-\Delta)(\Delta+\omega)(-\Delta+4 V+\omega)(\Delta-4 V+\omega)
\end{aligned}
$$

and a typical spectrum can be seen in Fig. 9a. In the homogeneous phase we make use of Eqs. (1719) and obtain the spectrum as

$$
S_{1}(v)=\frac{16 \kappa^{2} g^{8} \omega_{0}^{2}\left(\kappa^{2}+\omega_{0}^{2}\right)^{6}(\Delta-2 V)^{4}}{\Omega_{3} \Omega_{3}^{*}}
$$

with the abbreviated expressions

$$
\begin{aligned}
& \Omega_{3}=4 g^{4} \omega_{0}^{2}\left(\Omega_{31}+\Omega_{32}\right)+V^{2}\left(\kappa^{2}+\omega_{0}^{2}\right)^{2}\left(\Omega_{33}+\Omega_{34}+\Omega_{35}\right)+2 g^{2} V \omega_{0}\left(\kappa^{2}+\omega_{0}^{2}\right)\left(\Omega_{36}+\Omega_{37}+\Omega_{38}\right) \\
& \Omega_{31}=\Delta^{2}\left(\kappa^{2}+\omega_{0}^{2}\right)^{3}+\kappa^{6} \omega^{2}+2 i \kappa^{5} \omega^{3}-\kappa^{4}\left(\omega^{4}-3 \omega^{2} \omega_{0}^{2}\right)+4 i \kappa^{3} \omega^{3} \omega_{0}^{2}+\omega_{0}^{2}\left(\omega_{0}^{2}-\omega^{2}\right)\left(\omega^{2} \omega_{0}^{2}-16 g^{4}\right) \\
& \Omega_{32}=\kappa^{2} \omega_{0}^{2}\left(-16 g^{4}-2 \omega^{4}+3 \omega^{2} \omega_{0}^{2}\right)-2 i \kappa\left(16 g^{4} \omega \omega_{0}^{2}-\omega^{3} \omega_{0}^{4}\right) \\
& \Omega_{33}=-\kappa^{4}\left(8 \Delta g^{2} \omega_{0}+\omega^{4}-3 \omega^{2} \omega_{0}^{2}\right)+4 i \kappa^{3} \omega \omega_{0}\left(\omega^{2} \omega_{0}-4 \Delta g^{2}\right)+2 i \kappa \omega \omega_{0}^{2}\left(-8 \Delta g^{2} \omega_{0}-48 g^{4}+\omega^{2} \omega_{0}^{2}\right) \\
& \Omega_{34}=\kappa^{6} \omega^{2}+2 i \kappa^{5} \omega^{3}+\kappa^{2} \omega_{0}\left(8 \Delta g^{2}\left(\omega^{2}-2 \omega_{0}^{2}\right)-32 g^{4} \omega_{0}-2 \omega^{4} \omega_{0}+3 \omega^{2} \omega_{0}^{3}\right) \\
& \Omega_{35}=\omega_{0}^{2}\left(8 \Delta g^{2} \omega_{0}\left(\omega^{2}-\omega_{0}^{2}\right)+16 g^{4}\left(3 \omega^{2}-2 \omega_{0}^{2}\right)-\omega^{4} \omega_{0}^{2}+\omega^{2} \omega_{0}^{4}\right) \\
& \Omega_{36}=-8 \Delta g^{2} \omega_{0}\left(\kappa^{2}+\omega_{0}^{2}\right)^{2}+\Delta^{2}\left(\kappa^{2}+\omega_{0}^{2}\right)^{2}\left(\kappa^{2}+2 i \kappa \omega-\omega^{2}+\omega_{0}^{2}\right) \\
& \Omega_{37}=2\left(\kappa^{6} \omega^{2}+2 i \kappa^{5} \omega^{3}-\kappa^{4}\left(\omega^{4}-3 \omega^{2} \omega_{0}^{2}\right)+4 i \kappa^{3} \omega^{3} \omega_{0}^{2}+\omega_{0}^{2}\left(\omega_{0}^{2}-\omega^{2}\right)\left(\omega^{2} \omega_{0}^{2}-24 g^{4}\right)\right) \\
& \Omega_{38}=2\left(\kappa^{2} \omega_{0}^{2}\left(-24 g^{4}-2 \omega^{4}+3 \omega^{2} \omega_{0}^{2}\right)-2 i \kappa\left(24 g^{4} \omega \omega_{0}^{2}-\omega^{3} \omega_{0}^{4}\right)\right)
\end{aligned}
$$

depicted in Fig. 9d. We denote the cavity spectrum in the AFM+SR regime as $S_{4}(v)$. We solve Eqs. (12-15) numerically in the long-time limit and use Eq. (36) to determine the spectrum numerically, see Fig. 9b. Now we discuss the characteristic features and the behavior of the poles.

\section{Low-frequency pole structure for the case $(\kappa \neq 0, \gamma=0)$}

We begin our discussion with an analysis of the pole structure depicted in $S(v)$ in each phase. The frequency-resolved eigenenergies of the hybridized atom-cavity modes display a characteristic behaviour close to the phase transition as $g \rightarrow g_{\mathrm{c}, 1,2,3}$ in Fig. 2 . They can be obtained by solving 


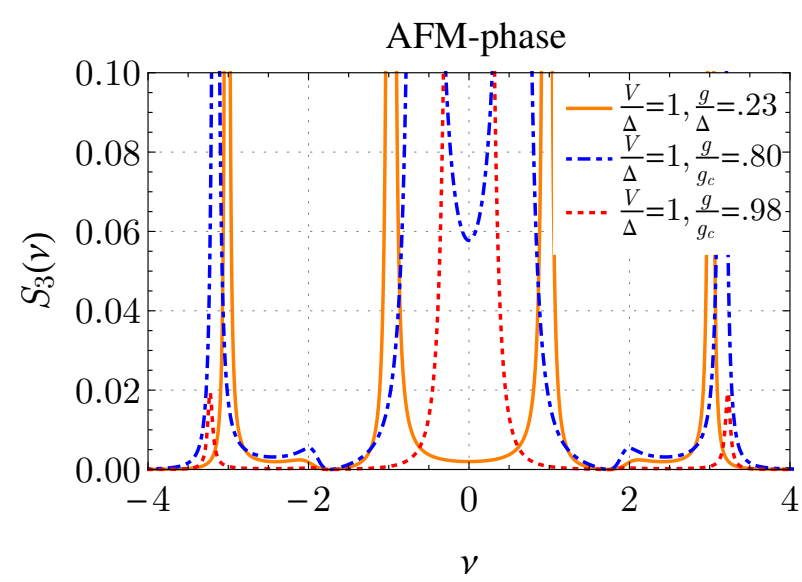

(a)

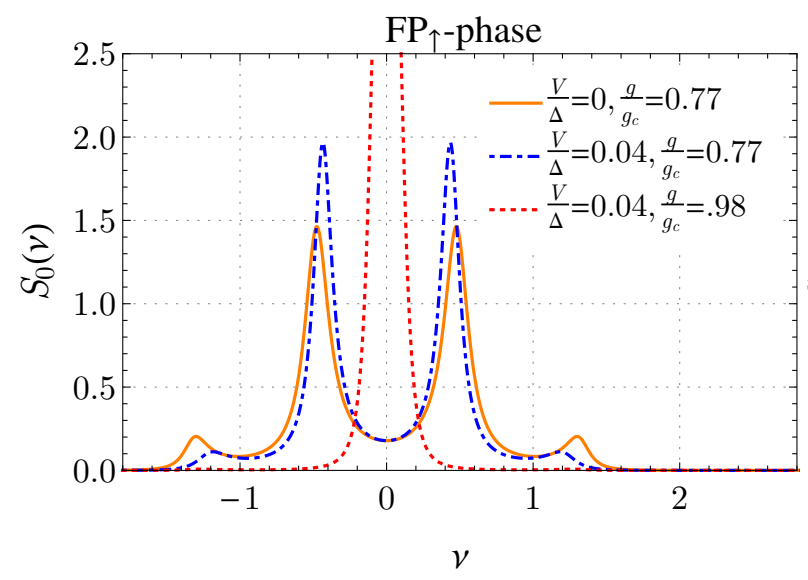

(c)

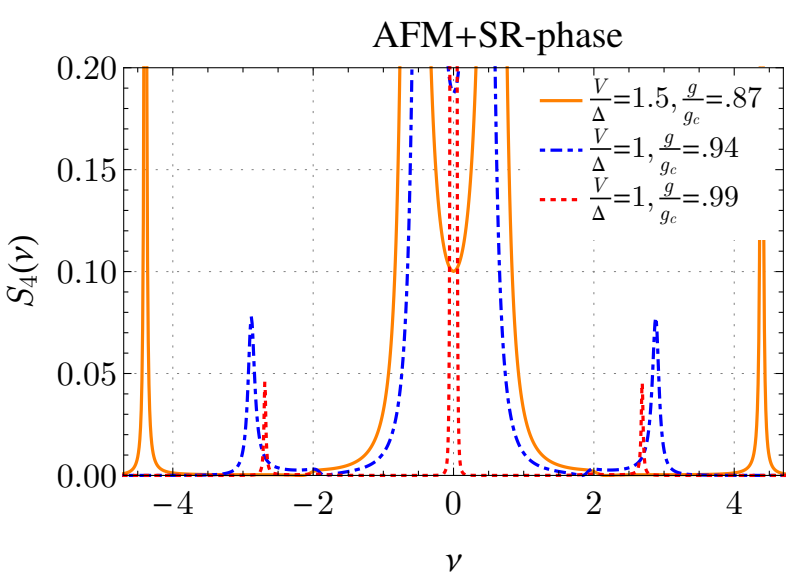

(b)

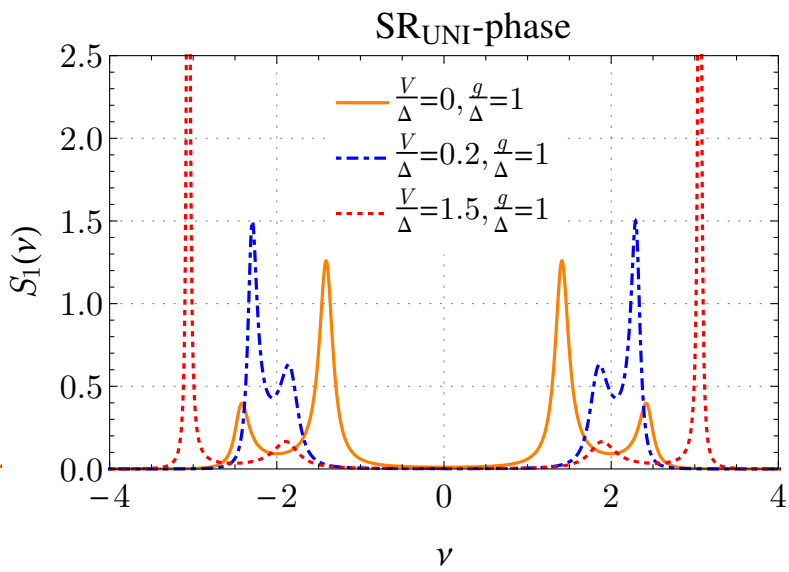

(d)

Figure 9. Typical cavity spectra for each of the four phases depicted in Fig. 2. In general, the resonances show the hybridised atom-cavity eigenenergies of the linearized dynamics given by Eq. (23). The spectrum exhibits two atomic and two photonic poles, when translational symmetry is broken, there are four atomic and two photonic branches. (a) Cavity spectrum in the plain antiferromagnetic phase. The broken translational symmetry is reflected in the appearance of an additional (Rydberg) resonance in the atomic-sector. (b) Cavity spectra in the regime of a broken $\mathbb{Z}_{2}$ and translational symmetry $T$. At the phase transition, translational symmetry is restored and the additional Rydberg-induced even-odd peak disappears. Close to the frequency at $v= \pm 2.0$ there are resonances with small but finite weight corresponding to the cavity frequency $\omega_{0} / \Delta=2.0$.(c) Cavity spectrum for the fully polarized phase $\mathrm{FP}_{\uparrow}$ with no photonic excitations $\langle a\rangle=0$. (d) Spectrum in the superradiant regime $\langle a\rangle \neq 0$ with translational symmetry $T$ still intact.

the characteristic equation $\operatorname{Det}\left[\boldsymbol{G}_{R}^{-1}(v)\right]=0$ in an expansion up to second-order in frequencies. 
We refer to the solutions of this equation by $\left(v_{1}, v_{2}\right)$. The low-frequency behaviour of the critical poles leading to the Dicke superradiance transition has already been established [69]. In terms of $\left(v_{1}, v_{2}\right)$ this means that both frequencies approach the imaginary axis and a single one vanishes at the phase-transition while the other obtains a finite imaginary part at $g=g_{c, 1}$ ('spectator' pole) set by the dissipation $\left(v_{2}\left(g=g_{c, 1}\right) \sim-i \kappa\right)$ emphasizing that the Dicke superradiance transition directly couples to the dissipation. When translational symmetry is broken, this transition mainly affects the atomic channel. As the photonic sector alone couples to a dissipative channel, we numerically observe that when translational symmetry is restored, there is no 'spectator' pole and the real and imaginary part of the low-frequency poles vanish together, see Fig. 3b.

In general there are either four or six poles in the cavity spectrum. In the former case these originate from two photon-branches and two atomic branches that are symmetrically arranged around the zero-frequency axis. We identify the branches by their $g \rightarrow 0$ limit in the fully polarised phase where the resonances settle at the bare frequencies given by $v_{\text {Atom }}= \pm \Delta$ and $v_{\text {Photon }}=$ $\pm \omega_{0}-i \kappa$. There are six poles when the translational symmetry in the atomic sector is broken. The additional poles reflect the even/odd imbalance of the system and are thus attributed to the Rydberg interaction, see Fig. 9b. This provides a clear feature to experimentally detect a phase with antiferromagnetic order.

We describe and depict the characteristic features of the cavity spectra for each phase of the meanfield phase diagram in Fig. 2 below. The cavity spectra in Fig. 9c (fully polarized $\mathrm{FP}_{\uparrow}$ ) and in Fig. 9d (superradiant phase) are well-known and derived in [40] in the $V \rightarrow 0$ limit. In the superradiant regime, an increasing Rydberg interaction $V$ shifts the atomic poles to higher energies whereas the peaks associated to the photonic branch settle around the cavity resonance at $v= \pm \omega_{0}=2.0|\Delta|$. In the AFM and the $(\mathrm{AFM}+\mathrm{SR})$ phase, the cavity spectra depicted in Fig. 9a and Fig. 9b exhibit the aforementioned even/odd sublattice peak that reflects the broken translational symmetry in the atomic sector. At the phase transition from the AFM into the (AFM+SR)-phase at $g=g_{\mathrm{c}, 3}$, the spectrum in Fig. 9a exhibits a pole with a purely imaginary frequency. The intensity under this finite-width peak at $v=0$ diverges, which indicates a macroscopic occupation of the cavity-mode $\langle a\rangle \neq 0$. In Fig. 9b, depicting the (AFM+SR) phase, one can see that as $g \rightarrow g_{\mathrm{c}, 2}$ one branch approaches the zero-frequency mode. In contrast to the superradiance transition, there is no 'spectator' pole and hence the width of the critical pole vanishes. At $g>g_{\mathrm{c}, 2}$ translational symmetry $T_{\text {lat }}$ is restored and the spectrum is given by Fig. $9 \mathrm{~d}$. 


\section{Result 3: Photon number oscillations}

1. Phase boundaries and order parameters with spontaneous emission $(\kappa \neq 0, \gamma \neq 0)$

As discussed in Sec. I D, allowing for spontaneous emission $(\gamma \neq 0)$ in addition to photon leakage $(\kappa \neq 0)$ has a dramatic influence on the phase diagram obtained from the behaviour of the meanfield master equations in the long-time limit. In comparison to the $\gamma=0$ case, the phase diagram is enriched by the presence of oscillatory and bistable phases, see Fig. 4 and Fig. 5. We first turn our attention to the case where there is a small amount of dissipation in the atomic channel $(\gamma=$ $0.01 \Delta$ ) to analyse its impact on the long-time limit behaviour of the steady-state phases depicted in Fig. 2. We observe (see Fig. 4) that allowing for a small amount of dissipation, there are no stable steady-states that involve a broken lattice symmetry $T_{\text {lat }}$. The only steady-states in the investigated $(V / \Delta, g / \Delta)$-plane is the empty atom-cavity system $\left(\mathrm{FP}_{\downarrow}\right)$ and a uniform superradiant phase $\left(\mathrm{SR}_{\mathrm{UNI}}\right)$. The remaining long-time limit behaviour is characterised by persistent oscillations that can be uniform $\left(\mathrm{SR}_{\mathrm{UNI}}-\mathrm{OSC}\right)$ or non-uniform $\left(\left(\mathrm{AFM}+\mathrm{SR}_{\mathrm{UNI}}\right)-\mathrm{OSC}\right)$. As the Rydberg interaction is conditioned on population in the upper state, the phase boundary of the empty atom-cavity system is independent of $V$. Formally, we obtain its phase boundary by inspecting the eigenvalues of the stability matrix corresponding to the fixed point $\left(\langle a\rangle=\left\langle\sigma^{x}\right\rangle=\left\langle\sigma^{y}\right\rangle=0,\left\langle\sigma^{z}\right\rangle=-1\right)$. The real part of at least one of the associated stability eigenvalues becomes positive when

$$
\gamma \kappa\left((\gamma+2 \kappa)^{2}+4 \Delta^{2}\right)^{2}-32 \Delta \lambda^{2} \omega_{0}(\gamma+2 \kappa)^{2}+8 \gamma \kappa \omega_{0}^{2}(\gamma-2 \Delta+2 \kappa)(\gamma+2(\Delta+\kappa))+16 \gamma \kappa \omega_{0}^{4}=0 .
$$

Solving for g yields,

$$
g_{\left(c r i t, F P_{\downarrow}\right)}=\frac{\sqrt{\gamma \kappa} \sqrt{\left((\gamma+2 \kappa)^{2}+4 \Delta^{2}\right)^{2}+8 \omega_{0}^{2}(\gamma-2 \Delta+2 \kappa)(\gamma+2(\Delta+\kappa))+16 \omega_{0}^{4}}}{4 \sqrt{2} \sqrt{\Delta \omega_{0}(\gamma+2 \kappa)^{2}}} .
$$

The transition into the stable $\mathrm{SR}_{\mathrm{UNI}}$-phase is discontinuous and we can solve Eqs. (12-15) in the long-time limit to obtain the photon condensate as

$$
\begin{aligned}
& |\langle a\rangle|^{2} \\
& =\frac{16 J^{3}(2 V-\Delta) \pm 2 \sqrt{J^{2}(\Delta+4 J)^{2}\left(4 J^{2}(\Delta-2 V)^{2}-2 \gamma^{2} J V-\gamma^{2} V^{2}\right)}-2 J^{2}\left(\gamma^{2}+2 \Delta(\Delta-2 V)\right)-\gamma^{2} J V}{16 J \omega_{0}(2 J+V)^{2}} \\
& =\frac{J^{2}(\Delta+4 J)(2 V-\Delta) \pm \sqrt{J^{4}(\Delta+4 J)^{2}(\Delta-2 V)^{2}}}{4 J \omega_{0}(2 J+V)^{2}}+O\left(\gamma^{2}\right)
\end{aligned}
$$




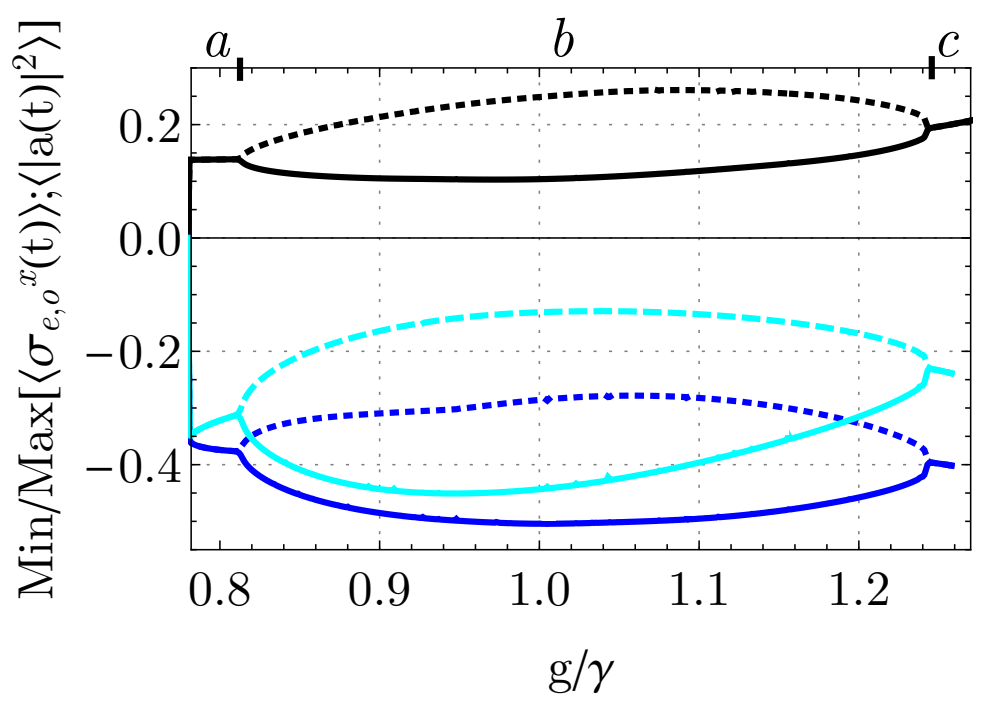

Figure 10. Amplitudes of the oscillations in the (AFM+SR)-OSC phase. Dashed (solid) blue lines show $\max (\min )\left[\left\langle\sigma_{e, o}^{x}(t)\right\rangle\right]$ and black lines show $\max (\min )\left[\left\langle|a(t)|^{2}\right\rangle\right]$. Data is obtained by extracting the minimum and maximum of the amplitudes of $\left\langle\sigma_{e, o}^{x}(t)\right\rangle$ and $\left\langle|a(t)|^{2}\right\rangle$ in a time interval chosen such that it contains several oscillations (if any are present) at long times. If the minimum and maximum coincide, the system settled into a steady state $(a, c)$ corresponding to the $(\mathrm{AFM}+\mathrm{SR})$ phase, otherwise the system is in the $(\mathrm{AFM}+\mathrm{SR})$-OSC limit cycle phase $(b)$. Close to the (AFM+SR)-phase, the amplitudes decay continuously. Parameters: $\left(\omega_{0} / \gamma=2.0, \Delta / \gamma=0.15, \kappa / \gamma=0.2, V / \gamma=1.8\right)$

where the coupling constant $J\left(\kappa, \omega_{0}\right)$ is given by Eq. (A9). Here the \pm sign indicates that there are two branches of which only one is stable. Stable solutions $\left(\mathrm{SR}_{\mathrm{UNI}}\right)$ are depicted in Fig. $4 \mathrm{a}$. In the limit of weak atomic noise, it can be seen from Eq. (43) that a stable solution to zeroth-order in $\gamma$ must satisfy $V>\Delta / 2$. The phase boundary between the two oscillating phases depicted in Fig. 4 is obtained by comparing the oscillation amplitudes (in Fig. 4b) on the even vs. the odd sublattices in the long-time limit that result from direct integration of Eqs. (12-15).

Next, we turn our attention to the features of the phase diagram depicted in Fig. 5 where losses in the atomic channel can be strong. We analyze the oscillations of both atomic and photonic components in the long-time limit by explicit integration of Eqs. (12-15). Numerically we find persistent oscillations close to the $(\mathrm{AFM}+\mathrm{SR})$ region that are different on the even/odd sublattice, see Fig. 6. We determine the phase boundary for the(AFM+SR)-OSC phase depicted in Fig. 5 by sampling initial conditions for the atomic components on the Bloch sphere and then integrating the set of Eqs. (12-15) directly. The phase boundary is set by the parameters $(\Delta / \gamma, g / \gamma)$ for which 


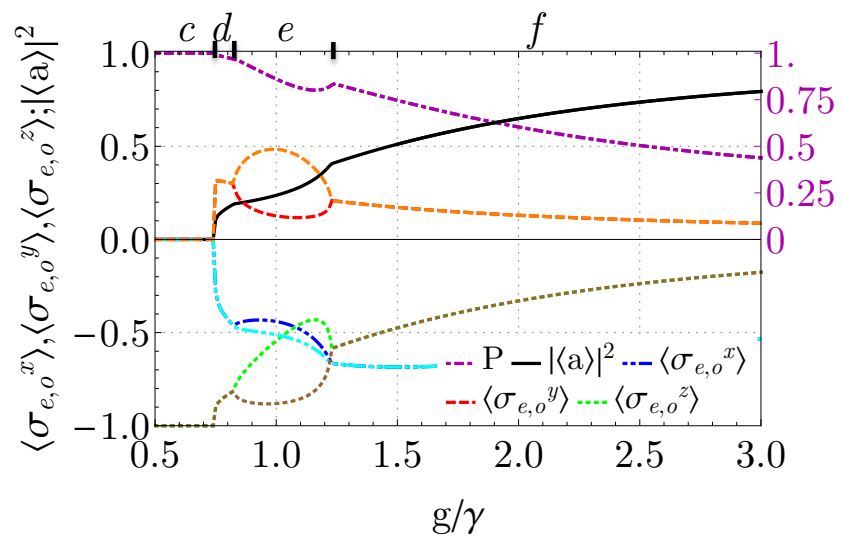

(a)

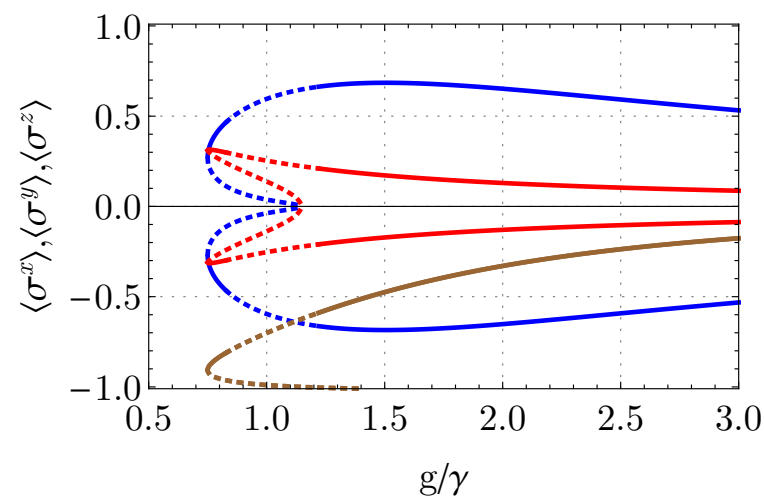

(b)

Figure 11. Non-monotonous behaviour of the order-parameters as the atom-light coupling $g / \gamma$ is varied. The system changes discontinuously from the empty atom-cavity system $\left(\mathrm{FP}_{\downarrow}, c\right)$ into a homogeneous phase $\left(\mathrm{SR}_{\mathrm{UNI}}, d\right)$ that becomes unstable towards an $(\mathrm{AFM}+\mathrm{SR}, e)$ phase that disappears again in favor for an $\left(\mathrm{SR}_{\mathrm{UNI}}, f\right)$ phase. On the right axis, the purity is shown that consistently decays, indicating the transition into a mixed state. (b) Stability analysis for homogeneous solutions as plotted in (a). (Unstable) stable, homogeneous solutions are plotted as (dotted) thick lines. The transition from the $\mathrm{FP}_{\downarrow}$ state into the $\mathrm{SR}_{\mathrm{UNI}}$ state is discontinuous if $V / \gamma>0$. Parameters: $\left(\omega_{0} / \gamma=2.0, \Delta / \gamma=-0.1, \kappa / \gamma=0.2, V / \gamma=1.8\right)$

the long-time limit is determined by the empty atom-cavity system $\left(\mathrm{FP}_{\downarrow}\right)$ for all initial conditions. In Fig. 10 we track the behaviour of the amplitude of the oscillations as a function of $g / \gamma$ and observe that the amplitudes decay continuously as the (AMR+SR) phase is approached. Numerically, we find no evidence that the (AFM+SR)-phase becomes unstable towards Hopf bifurcations meaning that stable limit cycles occur only outside the AFM+SR phase.

We continue our analysis by describing the behaviour of the magnetisations in the different domains of the phase diagram depicted in Fig. 5. In Fig. 11a we plot the magnetisation values in the steady-state for increasing the atom-light coupling $g / \gamma$. Starting in the empty atom-cavity, the system changes discontinuously into a $\mathrm{SR}_{\mathrm{UNI}}$ phase that soon after becomes unstable towards even/odd sublattice magnetisations $(\mathrm{AFM}+\mathrm{SR})$ that disappear again in favour for a re-entrance of the $\mathrm{SR}_{\mathrm{UNI}}$ phase. We depict the homogeneous solutions $\mathrm{SR}_{\mathrm{UNI}}$ and their stability in Fig. 11b. We find that for $V / \gamma>0$ the transition into the Dicke superradiance state is discontinuous.

We note that with $\gamma \neq 0$ the length of the semi-classical Bloch vector $\left\langle\boldsymbol{S}_{e, o}\right\rangle=\left(\left\langle\sigma_{e, o}^{x}\right\rangle,\left\langle\sigma_{e, o}^{y}\right\rangle,\left\langle\sigma_{e, o}^{z}\right\rangle\right)$ is not conserved any more and can shrink for increasing $g / \gamma$ values. In equilibrium systems, an 
increase in the coupling parameter should stabilize the order in the steady-state, here we instead observe a non-monotonous behaviour where the 'order parameters' decay again after having reached a maximum value. We illustrate this decay by plotting the purity $P=\operatorname{Tr}\left[\left(\rho_{e} \otimes \rho_{o}\right)^{2}\right]=\operatorname{Tr}\left[\rho_{e}^{2}\right] \operatorname{Tr}\left[\rho_{o}^{2}\right]$ of the density matrix alongside the magnetisations. Both quantities decay as $g / \gamma$ is increased. In the case of the purity $P$ this indicates the decay towards a purely mixed state.

The phase transitions in and out of the $(\mathrm{AFM}+\mathrm{SR})$ phases are continuous, whereas transitions from the empty atom-cavity system into the $\mathrm{SR}_{\mathrm{UNI}}$ phase are discontinuous for $V / \gamma>0$, see Fig. 11. On a mean-field level we observe bistabilities in the phase diagram depicted in Fig. 5. These could be induced by the nonlinearities in the mean-field master equations or can hint at non-trivial behaviour induced by fluctuations where the system in the long-time limit switches between the two steadystates predicted on a mean-field level [70]. Mostly, bistabilities occur with the empty atom-cavity system $\left(\mathrm{FP}_{\downarrow}\right)$. The corresponding stability line can be calculated analytically from the stability matrix and we find that it is independent of $V$ since the Rydberg-dressed interaction is conditioned on population in the excited state, see Eq. (41). The size of the (AFM+SR) region instead does depend on $V$.

\section{CONCLUSIONS AND FUTURE DIRECTIONS}

The point of this paper was to create a base case (model and approximate solution) for a large array of self-interacting atomic qubits coupled to a single-mode optical light field. Why do we believe this is needed? Because there is an increasing number of experimental platforms ranging from ultracold atoms in optical cavities, superconducting circuits, photonic pulses travelling through Rydberg gases to nano-photonic crystals, seeking to scale up the number of qubits and interface them with photons. Qubit-qubit interactions can be wanted -to mediate photon-photon nonlinearities for example- or stray, in which case they would be seen to dephase a collective coupling of a set of qubits to photons. In the face of the considerable complexity these systems generate -large number of quantum spins, fluctuating light fields, non-equilibrium aspects- our simple model has yielded some experimentally directly testable predictions: coexistence of magnetic translation symmetry breaking and superradiance, a new even-odd collective mode in the cavity spectrum, and intriguing, oscillating solutions for both, the spin components and a coherent photon field. Present-day technology with Rydberg-dressed spin lattices in optical cavities should be able to check and refine these results. 
Unfortunately, we were not able to solve even our simple model exactly; the Rydberg-mediated nearest-neighbour interaction does induce non-trivial quantum fluctuations (centered around the even-odd modulation momentum $(\pi, \pi)$ ) between the spins. Our non-equilibrium mean-field ansatz for the density matrix kept track of only the expectation values of the spin components on the even and odd sub-lattices, and the photon field, respectively. We are not aware of a developed technique, which can capture quantum fluctuations for large, far-from-equilibrium quantum spin systems coupled to the (potentially large) Hilbert space of one or multiple photon modes. Promising efforts in this direction invoke a fermion representation of the quantum spins on the closed-time Keldysh contour ([71, 72] and references therein); this then, in principle, allows to leverage over diagrammatic techniques well-developed for ground state fermions. Work in this direction is underway.

Particularly promising physical set-ups to study the interplay of interacting qubits with light in the future are nano-photonic and one-dimensional quantum-optical systems [35, 36, 73-78], in which huge effects from even small qubit-qubit interactions can be expected. Moreover, these systems typically contain an (infinite) continuum of photon modes significantly enriching the complexity of the photonic Hilbert space at one's disposal. The same is true for optical resonators in multi-mode operation $[30,79,80]$ making them also interesting targets for further explorations.

\section{ACKNOWLEDGMENTS}

We thank A. Glätzle, C. Kollath, and P. Zoller for good discussions and further acknowledge helpful remarks by T. Pohl, P. Rabl, H. Ritsch, and J. Zeiher during the Quantum Optics 2016 conference. JG thanks the Department of Physics at Harvard University for hospitality, where this research was partly carried out (during the first half of 2015). We are grateful to M. D. Lukin for discussions on related topics. We also thank the Sachdev Group for sharing their bibliography style file. This work was supported by the Leibniz Prize of A. Rosch, by the Harvard-MIT Center for Ultracold Atoms (CUA), by the Multidisciplinary University Research Initiative (MURI), and by the German Research Foundation (DFG) through the Institutional Strategy of the University of Cologne within the German Excellence Initiative (ZUK 81). 


\section{Appendix A: Mean-field solution of the $\mathbf{T}=\mathbf{0}$ equilibrium spin model}

In this section, we analyze Hamiltonians $H_{\text {spin-light }}$ Eq. (2) and $H_{\text {spin }}$ Eq. (1) within a (standard) equilibrium mean-field theory for spins. We will find similar phases to those in Fig. 2 upon identifying one of the spin-spin interaction constants with cavity parameters, somewhat surprisingly including the photon decay $\kappa$. The deeper reason for this is that with the counter-rotating terms, in the atom-light coupling the excitation number is stabilized despite the loss rates. Accordingly, the non-equilibrium steady-state phase in the long-time limit are then qualitatively similar to the ground state phases. Dynamics and statistics (effective temperature) of the full non-equilibrium system, however, remain qualitatively drastically different.

First, we integrate out the quadratic photon terms which yields an effective Hamiltonian that features a ferromagnetic all-to-all atom-atom coupling $J$. The connection to the non-equilibrium system is then made explicit by letting $J$ depend on $\kappa$ as pointed out below. The Hamiltonian we consider is written as

$$
\tilde{H}=-\frac{J}{N} \sum_{i j} \sigma_{i}^{x} \sigma_{j}^{x}-\frac{\Delta}{2} \sum_{i} \sigma_{i}^{z}+\frac{1}{2} V \sum_{\langle\ell m\rangle}^{N} \sigma_{\ell}^{e e} \sigma_{m}^{e e}
$$

Where the $1 / 2$ in front of the Rydberg interaction avoids overcounting. We cast the last terms into a spin-language with the replacement $\sigma_{\ell}^{e e}=1 / 2\left(1+\sigma_{\ell}^{z}\right)$. We decouple the interaction terms in meanfield theory by expanding the operators around their mean-value to linear order in fluctuations. We neglect all second-order fluctuation terms and write the effective spin-Hamiltonian in a form that resembles the interaction of the spin-variables with an effective, local magnetic field that needs to be determined self-consistently and represents the mean field from the neighbouring spins. Ignoring constant energy shifts, the full mean-field Hamiltonian assuming $d=2$-spatial dimensions is given as

$$
\tilde{H}^{\mathrm{MF}}=-V \frac{N}{2}\left\langle\sigma_{\text {even }}^{z}\right\rangle\left\langle\sigma_{\text {odd }}^{z}\right\rangle+N J\left\langle\sigma^{x}\right\rangle^{2}+\sum_{i \in \text { even }} \boldsymbol{B}^{\text {even }} \boldsymbol{\sigma}_{i}+\sum_{j \in \text { odd }} \boldsymbol{B}^{\text {odd }} \boldsymbol{\sigma}_{j}
$$

Here, we have already accounted for an even/odd sub-lattice asymmetry in the $z$-components. We use the vector of Pauli matrices as $\sigma=\left(\sigma^{x}, \sigma^{y}, \sigma^{z}\right)^{T}$ and define the local magnetic fields as

$$
\boldsymbol{B}^{\text {even/odd }}=\left(\left[\left\langle\sigma_{\text {odd } / \text { even }}^{z}\right\rangle V+(V-\Delta / 2)\right] \hat{z}+2 J\left\langle\sigma^{x}\right\rangle \hat{\boldsymbol{x}}\right)
$$

We evaluate the partition sum

$$
Z=\operatorname{Tr}\left[e^{-\beta \tilde{H}^{\mathrm{MF}}}\right]=2^{N}\left[\cosh \left(\left|\boldsymbol{B}^{\text {even }}\right|\right) \cosh \left(\left|\boldsymbol{B}^{\text {odd }}\right|\right)\right]^{N / 2} \exp \left[V \frac{N}{2} \beta\left\langle\sigma_{\text {even }}^{z}\right\rangle\left\langle\sigma_{\text {odd }}^{z}\right\rangle-N \beta J\left\langle\sigma^{x}\right\rangle^{2}\right]
$$


to obtain the self-consistency equations for the order-parameters

$$
\begin{gathered}
\phi=\frac{\left\langle\sigma_{\text {even }}^{x}\right\rangle+\left\langle\sigma_{\text {odd }}^{x}\right\rangle}{2}=\frac{1}{2} \tanh \left(\beta\left|\boldsymbol{B}^{\text {odd }}\right|\right) \frac{B_{x}^{\text {odd }}}{\left|\boldsymbol{B}^{\text {odd }}\right|}+\frac{1}{2} \tanh \left(\beta\left|\boldsymbol{B}^{\text {even }}\right|\right) \frac{B_{x}^{\text {even }}}{\left|\boldsymbol{B}^{\text {even }}\right|} \\
\rho=\frac{\left\langle\sigma_{\text {even }}^{z}\right\rangle-\left\langle\sigma_{\text {odd }}^{z}\right\rangle}{2}=\frac{1}{2} \tanh \left(\beta\left|\boldsymbol{B}^{\text {even }}\right|\right) \frac{B_{z}^{\text {even }}}{\left|\boldsymbol{B}^{\text {even }}\right|}-\frac{1}{2} \tanh \left(\beta\left|\boldsymbol{B}^{\text {odd }}\right|\right) \frac{B_{z}^{\text {odd }}}{\left|\boldsymbol{B}^{\text {odd }}\right|} \\
\rho_{0}=\frac{\left\langle\sigma_{\text {even }}^{z}\right\rangle+\left\langle\sigma_{\text {odd }}^{z}\right\rangle}{2}=\frac{1}{2} \tanh \left(\beta\left|\boldsymbol{B}^{\text {even }}\right|\right) \frac{B_{z}^{\text {even }}}{\left|\boldsymbol{B}^{\text {even }}\right|}+\frac{1}{2} \tanh \left(\beta\left|\boldsymbol{B}^{\text {odd }}\right|\right) \frac{B_{z}^{\text {odd }}}{\left|\boldsymbol{B}^{\text {odd }}\right|}
\end{gathered}
$$

Where $\rho$ is the staggered magnetisation and $\rho_{0}$ is the average magnetisation in the $z$ direction. The magnetic order parameter $\phi$ measures the magnetisation in $x$-direction and $\beta=1 / T$ is the inverse temperature. We denote the free energy per spin in the zero temperature limit $T \rightarrow 0$ as

$$
\begin{aligned}
f=\left.\frac{F}{N}\right|_{T \rightarrow 0} & =-\left.\frac{T}{N} \log (Z)\right|_{T \rightarrow 0}=\frac{1}{2} V\left(\rho^{2}-\rho_{0}^{2}\right)+J \phi^{2} \\
& -\frac{1}{2}\left(\sqrt{\left(V\left(\rho_{0}-\rho\right)+V-\frac{\Delta}{2}\right)^{2}+4 J^{2} \phi^{2}}+\sqrt{\left(V\left(\rho+\rho_{0}\right)+V-\frac{\Delta}{2}\right)^{2}+4 J^{2} \phi^{2}}\right)
\end{aligned}
$$

We can determine the zero-temperature phase-diagram by solving the coupled set of Eqs. (A5 - A7) numerically and retain only the solutions with the lowest free-energy according to Eq. (A8). We obtain the splitting in the $\left(\left\langle\sigma_{\text {even }}^{x}\right\rangle,\left\langle\sigma_{\text {odd }}^{x}\right\rangle\right)$ components by using Eq. (16). We find that we can map the equilibrium phase-diagram to the phase diagram obtained by calculating the non-equilibrium steady-states (see Fig. 2) if we identify the ferromagnetic exchange coupling as

$$
J(g, \kappa)=\frac{g^{2} \omega_{0}}{\omega_{0}^{2}+\kappa^{2}}
$$

This coupling is inferred from solving for the steady-state values of the photons (see Eq. (15)) which is given as $g\left(\langle a\rangle+\left\langle a^{\dagger}\right\rangle\right)=-\frac{1}{2} g\left\langle\sigma_{\text {even }}^{x}+\sigma_{\text {odd }}^{x}\right\rangle\left(\frac{g}{\omega-i \kappa}+\frac{g}{\omega+i \kappa}\right) \propto J(g, \kappa)$. Allowing the interpretation that the photonic losses with rate $\kappa$ weaken the atom-atom couplings.

\section{Appendix B: Transformation of fully time-dependent model into rotating frame}

Here, we detail calculations where we derive how the parameters of the Hamiltonians $H_{\text {spin-light }}$ given by Eq. (2) and $H_{\text {spin }}$ given by Eq. (1) are related to tunable laser parameters that result from 
the optical implementation shown in Fig. 7. The Hamiltonian we consider is of the form

$$
\begin{aligned}
H & =H_{\text {cav }}+H_{\text {atoms }}+H(t)_{\text {pump }}+H_{\text {atom-light }}+H_{\text {atom-atom }} \\
H_{\text {cav }} & =\omega_{0} a^{\dagger} a \\
H_{\text {atoms }} & =\sum_{\ell=1}^{N} \omega_{d}|d\rangle_{\ell}\left\langle d\left|+\omega_{e}\right| e\right\rangle_{\ell}\left\langle e\left|+\omega_{\text {Ryd }}\right| \mathrm{Ryd}\right\rangle_{\ell}\left\langle\mathrm{Ryd}\left|+\omega_{1}\right| 1\right\rangle_{\ell}\langle 1| \\
H_{\text {pump }}(t) & =\sum_{\ell=1}^{N} \frac{\Omega_{\ell}}{2} e^{-i \omega_{\Delta e} t}|e\rangle_{\ell}\left\langle 1\left|+\frac{\Omega_{d}}{2} e^{-i \omega_{\Delta d} t}\right| d\right\rangle_{\ell}\left\langle 0\left|+\frac{\Omega_{\mathrm{Ryd}}}{2} e^{-i \omega_{\Delta r} t}\right| \mathrm{Ryd}\right\rangle_{\ell}\langle 1|+\text { h.c. } \\
H_{\text {atom-light }} & =\sum_{\ell=1}^{N}\left(g_{d}|d\rangle_{\ell}\left\langle 1\left|+g_{e}\right| e\right\rangle_{\ell}\langle 0|\right) a+\text { h.c. } \\
H_{\text {atom-atom }} & =\sum_{\langle\ell m\rangle} V_{\ell m}\left(|\mathrm{Ryd}\rangle_{\ell}\langle\mathrm{Ryd}|\right)\left(|\mathrm{Ryd}\rangle_{m}\langle\mathrm{Ryd}|\right)
\end{aligned}
$$

The frequencies $\left(\omega_{d}, \omega_{e}, \omega_{\mathrm{Ryd}}, \omega_{1}\right)$ refer to the atomic levels labelled by the sequence $(d, e, \operatorname{Ryd}, 1)$ and are measured relative to the atomic level $|0\rangle$. Correspondingly, the frequencies $\left(\omega_{\Delta d}, \omega_{\Delta e}, \omega_{\Delta r}\right)$ refer to the laser frequencies of the pump-terms. Here, $\omega_{0}$ denotes the bare cavity resonance. We have assumed homogeneous pumping of the atoms from the side $\Omega_{(d, e) ; \ell} \approx \Omega_{(d, e)}$ and a homogeneous coupling of the light field to the atoms $g_{(d, e) ; \ell} \approx g_{(d, e)}$. We eliminate the explicit timedependence by switching into a rotating frame such that the new Hamiltonian reads

$$
\tilde{H}=U^{\dagger} H_{0} U-U^{\dagger} i \partial t U
$$

where the Hamiltonian $H_{0}$ is given as

$$
\begin{aligned}
U(t) & =\exp \left(-i H_{0} t\right) \\
H_{0} & =\left(\omega_{\Delta d}-\omega_{1}^{\prime}\right) a^{\dagger} a+\sum_{\ell=1}^{N}\left[\left(\omega_{\Delta e}+\omega_{1}^{\prime}\right)|e\rangle_{\ell}\left\langle e\left|+\omega_{\Delta d}\right| d\right\rangle_{\ell}\left\langle d\left|+\omega_{1}^{\prime}\right| 1\right\rangle_{\ell}\left\langle 1\left|+\left(\omega_{\mathrm{Ryd}}+\omega_{1}^{\prime}\right)\right| \mathrm{Ryd}\right\rangle_{\ell}\langle\mathrm{Ryd}|\right]
\end{aligned}
$$

Cross coupling lasers need to be tuned such that they are strongly detuned from the levels $(|d\rangle,|e\rangle)$ which can then be eliminated adiabatically. Under the condition $\left|\Delta_{d, e}\right| \gg \kappa, \gamma, \Omega_{d, e}$, the dynamics 
of the system are now described by an effective Hamiltonian $\tilde{H}=\tilde{H}_{\mathrm{Ryd}}+\tilde{H}_{10}+\tilde{H}_{\mathrm{L}}$ :

$$
\begin{aligned}
\tilde{H}_{L} & =\sum_{\ell=1}^{N} \frac{\Omega_{\mathrm{Ryd}}}{2}\left(|\mathrm{Ryd}\rangle_{\ell}\langle 1|+| 1\rangle_{\ell}\langle\mathrm{Ryd}|\right) \\
\tilde{H}_{\mathrm{Ryd}} & =-\Delta_{\mathrm{Ryd}} \sum_{\ell=1}^{N}|\mathrm{Ryd}\rangle_{\ell}\langle\mathrm{Ryd}|+\sum_{\langle\ell m\rangle} V_{\ell m}\left(|\mathrm{Ryd}\rangle_{\ell}\langle\mathrm{Ryd}|\right)\left(|\mathrm{Ryd}\rangle_{m}\langle\mathrm{Ryd}|\right) \\
\tilde{H}_{10}= & \omega_{a} a^{\dagger} a+\sum_{\ell=1}^{N}\left[\left(\Delta_{1}+\frac{\Omega_{e}^{2}}{4 \Delta_{e}}\right)|1\rangle_{\ell}\left\langle 1\left|+\frac{\Omega_{d}}{4 \Delta_{d}}\right| 0\right\rangle_{\ell}\langle 0|+\frac{1}{2}\left(\frac{g_{e} \Omega_{e}}{\Delta_{e}}|0\rangle_{\ell}\left\langle 1\left|a^{\dagger}+\frac{g_{d} \Omega_{d}}{\Delta_{d}}\right| 1\right\rangle_{\ell}\langle 0| a^{\dagger}+\text { h.c. }\right)\right. \\
& \left.+\left(\frac{g_{e}^{2}}{\Delta_{e}}|0\rangle_{\ell}\left\langle 0\left|+\frac{g_{d}^{2}}{\Delta_{d}}\right| 1\right\rangle_{\ell}\langle 1|\right) a^{\dagger} a\right]
\end{aligned}
$$

where we have used the following frequencies

$$
\begin{aligned}
\omega_{a} & =\omega_{0}-\left(\omega_{\Delta d}-\omega_{1}^{\prime}\right), \\
\Delta_{\mathrm{Ryd}} & =-\left[\omega_{\mathrm{Ryd}}-\left(\omega_{\Delta r}+\omega_{1}^{\prime}\right)\right] \\
\Delta_{1} & =\omega_{1}-\omega_{1}^{\prime}, \\
2 \omega_{1}^{\prime} & =\omega_{\Delta d}-\omega_{\Delta e} .
\end{aligned}
$$

In a next step, high-lying Rydberg states are admixed to the ground-states $|1\rangle_{\ell}$ to realise a Rydbergdressed interaction between the states $|\tilde{1}\rangle=|1\rangle+\frac{\Omega_{\mathrm{Ryd}}}{2 \Delta_{\mathrm{Ryd}}}|\mathrm{Ryd}\rangle+O\left(\frac{\Omega_{\mathrm{Ryd}}}{2 \Delta_{\mathrm{Ryd}}}\right)$. Typically, two-body Born-Oppenheimer potentials as a function of the distance $r_{i j}$ between two Rydberg levels are obtained by diagonalising Hamiltonians of the form $H_{L}+H_{\mathrm{Ryd}}$ in a two-atom basis [65]. A detailed calculation that includes coupling to the complicated level structure is thus highly non-trivial. Focusing on the weak-dressing regime $\Omega_{\text {Ryd }} / \Delta_{\text {Ryd }} \ll 1$ and red-detuning of the dressing laser we follow the many-body perturbation expansion performed in Ref. [64] to obtain the effective Hamiltonian for the Rydberg part to leading order in the corrections

$$
\begin{aligned}
\tilde{H}_{\mathrm{Ryd}} & =-\frac{\Omega_{\mathrm{Ryd}}^{2}}{4 \Delta_{\mathrm{Ryd}}} \sum_{\ell=1}^{N}|\tilde{1}\rangle_{\ell}\langle\tilde{1}|+\frac{1}{2}\left(\frac{\Omega_{\mathrm{Ryd}}}{2 \Delta_{\mathrm{Ryd}}}\right)^{4} \sum_{i \neq j} \frac{C_{6}}{r_{i j}+R_{c}^{6}}\left(|\tilde{1}\rangle_{i}\langle\tilde{1}|\right)\left(|\tilde{1}\rangle_{j}\langle\tilde{1}|\right) \\
& =-\frac{\Omega_{\mathrm{Ryd}}^{2}}{4 \Delta_{\mathrm{Ryd}}} \sum_{\ell=1}^{N}|\tilde{1}\rangle_{\ell}\langle\tilde{1}|+\frac{1}{2} \sum_{i \neq j} V_{\mathrm{ij}}^{\mathrm{eff}}\left(|\tilde{1}\rangle_{i}\langle\tilde{1}|\right)\left(|\tilde{1}\rangle_{j}\langle\tilde{1}|\right)
\end{aligned}
$$

It can be seen that the dressed states $|\tilde{1}\rangle$ acquire additional light-shifts $\sim \Omega_{\text {Ryd }}^{2} /\left(4 \Delta_{\text {Ryd }}\right)$ and the Rydberg potential is tunable by changing $\left(\Omega_{\mathrm{Ryd}}, \Delta_{\mathrm{Ryd}}\right)$. Here, $V_{i j}^{\text {eff }}$ and $R_{c}$ are defined in Eq. (10). 
We now replace $|1\rangle$ with the dressed Rydberg state $|\tilde{1}\rangle$ everywhere in Eq. (B12). With

$$
\begin{aligned}
& \sum_{\ell=1}^{N}|1\rangle_{\ell}\langle 1|=\frac{1}{2} \sum_{\ell=1}^{N}\left(|1\rangle_{\ell}\langle 1|-| 0\rangle_{\ell}\langle 0|+N\right)=\frac{1}{2} \sum_{\ell=1}^{N} \sigma_{\ell}^{z}+\frac{N}{2} \\
& \sum_{\ell=1}^{N}|0\rangle_{\ell}\langle 0|=-\frac{1}{2} \sum_{\ell=1}^{N}\left(|1\rangle_{\ell}\langle 1|-| 0\rangle_{\ell}\langle 0|-N\right)=-\frac{1}{2} \sum_{\ell=1}^{N} \sigma_{\ell}^{z}+\frac{N}{2}
\end{aligned}
$$

the Hamiltonian is now cast into the form:

$$
\begin{aligned}
\tilde{H}= & a^{\dagger} a\left[\frac{N}{2}\left(\frac{g_{e}^{2}}{\Delta_{e}}+\frac{g_{d}^{2}}{\Delta_{d}}\right)+\omega_{a}\right] \\
& +\sum_{\ell=1}^{N} \sigma_{\ell}^{z} \frac{1}{2}\left[\left(\frac{\Omega_{d}^{2}}{4 \Delta_{d}}-\frac{\Omega_{e}^{2}}{4 \Delta_{e}}\right)+\Delta_{1}-\frac{\Omega_{\mathrm{Ryd}}^{2}}{4 \Delta_{\mathrm{Ryd}}}+\frac{1}{2} \sum_{m(m \neq \ell)}^{N} V_{m \ell}^{\mathrm{eff}}\right]+\sum_{\ell=1}^{N} \sigma_{\ell}^{z} \frac{1}{2} \frac{1}{N}\left(\frac{g_{d}^{2}}{\Delta_{d}}-\frac{g_{e}^{2}}{\Delta_{e}}\right) a^{\dagger} a \\
& +\sum_{\ell=1}^{N}\left[\frac{\lambda_{d}}{\sqrt{N}}\left(\sigma_{\ell}^{+} a^{\dagger}+\sigma_{\ell}^{-} a\right)+\frac{\lambda_{e}}{\sqrt{N}}\left(\sigma_{\ell}^{+} a+\sigma_{\ell}^{-} a^{\dagger}\right)\right] \\
& +\frac{1}{2} \sum_{i \neq j} V_{\mathrm{ij}}^{\mathrm{eff}} \frac{\sigma_{i}^{z}}{2} \frac{\sigma_{j}^{z}}{2}
\end{aligned}
$$

with effective spin-photon couplings (set equal and denoted by $g$ in Eq. (9))

$$
\lambda_{d, e}=\sqrt{N} \frac{g_{d, e} \Omega_{d, e}}{2 \Delta_{d, e}}
$$

To generate AFM ordering it is advantageous for the effective longitudinal field corresponding to the second term in the first line in Eq. (B17) to be negative. We analyse typical orders of magnitudes. The hyperfine structure splitting in the ground state manifold is $\omega_{1}=2 \pi \times 6.835 \mathrm{GHz}$. Typically the cavity-assisted Raman transitions are achieved by coupling to the first excited state manifold that is split into a fine-structure $5^{2} P_{1 / 2}$ with $F^{\prime}=2$ and $F^{\prime}=1$ that for this choice is on the order of $812 \mathrm{MHz}$. The external driving lasers are separated by approximately twice the ground-state hyperfine splitting $\omega_{1^{\prime}}=\frac{1}{2}\left(\omega_{\Delta d}-\omega_{\Delta e}\right) \sim \omega_{1}$ such that $\Delta_{1}=\omega_{1}-\omega_{1^{\prime}} \sim \mathrm{MHz}$. For weakly admixing the Rydberg state to the groundstate manifold $|\uparrow\rangle$ the detuning from the Rydberg state $\Delta_{\text {Ryd }}$ must satisfy $\Omega_{\text {Ryd }} \ll \Delta_{\text {Ryd }}$. Typical Rabi frequencies for the drive to the Rydberg level are $\Omega_{\text {Ryd }} \sim \mathrm{MHz}$. The detuning from the Rydberg level now has a frequency component $\omega_{1^{\prime}}$ from the Raman-scheme: $\Delta_{\mathrm{Ryd}}=-\left[\omega_{r}-\left(\omega_{\Delta r}+\omega_{1^{\prime}}\right)\right]$. This can take the usual detuning $\Delta_{\mathrm{Ryd}}$ far above the MHz regime which makes $\Delta_{\mathrm{Ryd}} \ggg \Omega_{\mathrm{Ryd}}$. The longitudinal field $\left(\Delta_{1}-\frac{\Omega_{\mathrm{Ryd}}^{2}}{4 \Delta_{\mathrm{Ryd}}}\right)$ for $\Omega_{d}=\Omega_{e}$ and $\Delta_{d}=\Delta_{e}$ is in the $\mathrm{MHz}$ range and can in principle be tuned positive and negative. 
[1] I. Bloch, J. Dalibard, and S. Nascimbene, "Quantum simulations with ultracold quantum gases," Nat Phys 8, 267 (2012).

[2] C. Gardiner and P. Zoller, The Quantum World of Ultra-Cold Atoms and Light Book II: The Physics of Quantum-Optical Devices, 1st ed. (World Scientific, 2015).

[3] R. Landig, L. Hruby, N. Dogra, M. Landini, R. Mottl, T. Donner, and T. Esslinger, "Quantum phases from competing short- and long-range interactions in an optical lattice,” Nature 532, 476 (2016).

[4] J. Klinder, H. Keßler, M. R. Bakhtiari, M. Thorwart, and A. Hemmerich, "Observation of a superradiant mott insulator in the dicke-hubbard model," Phys. Rev. Lett. 115, 230403 (2015).

[5] J. Zeiher, R. van Biijnen, P. Schauss, S. Hild, J. Choi, T. Pohl, I. Bloch, and C. Gross, "Many-body interferometry of a rydberg-dressed spin lattice," arXiv:1602.06313 (2016).

[6] T. Peyronel, O. Firstenberg, Q.-Y. Liang, S. Hofferberth, A. V. Gorshkov, T. Pohl, M. D. Lukin, and V. Vuletic, "Quantum nonlinear optics with single photons enabled by strongly interacting atoms," Nature 488, 57 (2012).

[7] Electromagnetically Induced Transparency.

[8] F. Piazza and P. Strack, "Umklapp superradiance with a collisionless quantum degenerate fermi gas," Phys. Rev. Lett. 112, 143003 (2014).

[9] P. Strack and S. Sachdev, "Dicke quantum spin glass of atoms and photons," Phys. Rev. Lett. 107, $277202(2011)$.

[10] S. Gopalakrishnan, B. L. Lev, and P. M. Goldbart, "Frustration and glassiness in spin models with cavity-mediated interactions," Phys. Rev. Lett. 107, 277201 (2011).

[11] H. Habibian, A. Winter, S. Paganelli, H. Rieger, and G. Morigi, "Bose-glass phases of ultracold atoms due to cavity backaction," Phys. Rev. Lett. 110, 075304 (2013).

[12] M. Buchhold, P. Strack, S. Sachdev, and S. Diehl, "Dicke-model quantum spin and photon glass in optical cavities: Nonequilibrium theory and experimental signatures," Phys. Rev. A 87, 063622 (2013).

[13] M. Müller, P. Strack, and S. Sachdev, "Quantum charge glasses of itinerant fermions with cavitymediated long-range interactions,” Phys. Rev. A 86, 023604 (2012).

[14] S. F. Caballero-Benitez and I. B. Mekhov, "Bond order via light-induced synthetic many-body interactions of ultracold atoms in optical lattices," arXiv:1604.02563 (2016). 
[15] Y. Deng, J. Cheng, H. Jing, and S. Yi, "Bose-einstein condensates with cavity-mediated spin-orbit coupling,” Phys. Rev. Lett. 112, 143007 (2014).

[16] J.-S. Pan, X.-J. Liu, W. Zhang, W. Yi, and G.-C. Guo, “Topological superradiant states in a degenerate fermi gas,” Phys. Rev. Lett. 115, 045303 (2015).

[17] C. Kollath, A. Sheikhan, S. Wolff, and F. Brennecke, "Ultracold fermions in a cavity-induced artificial magnetic field,” Phys. Rev. Lett. 116, 060401 (2016).

[18] W. Zheng and N. R. Cooper, "Superradiance induced particle flow via dynamical gauge coupling," arXiv:1604.06630 (2016).

[19] S. Trotzky, P. Cheinet, S. Fölling, M. Feld, U. Schnorrberger, A. M. Rey, A. Polkovnikov, E. A. Demler, M. D. Lukin, and I. Bloch, "Time-resolved observation and control of superexchange interactions with ultracold atoms in optical lattices," Science 319, 295 (2008).

[20] J. Simon, W. S. Bakr, R. Ma, M. E. Tai, P. M. Preiss, and M. Greiner, "Quantum simulation of antiferromagnetic spin chains in an optical lattice,” Nature 472, 307 (2011).

[21] T. Lahaye, C. Menotti, L. Santos, M. Lewenstein, and T. Pfau, "The physics of dipolar bosonic quantum gases," Reports on Progress in Physics 72, 126401 (2009).

[22] B. Yan, S. A. Moses, B. Gadway, J. P. Covey, K. R. A. Hazzard, A. M. Rey, D. S. Jin, and J. Ye, “Observation of dipolar spin-exchange interactions with lattice-confined polar molecules," Nature 501, 521 (2013).

[23] S. Baier, M. J. Mark, D. Petter, K. Aikawa, L. Chomaz, Z. Cai, M. Baranov, P. Zoller, and F. Ferlaino, "Extended bose-hubbard models with ultracold magnetic atoms," Science 352, 201 (2016).

[24] R. M. W. van Bijnen and T. Pohl, "Quantum magnetism and topological ordering via rydberg dressing near förster resonances,” Phys. Rev. Lett. 114, 243002 (2015).

[25] A. W. Glaetzle, M. Dalmonte, R. Nath, I. Rousochatzakis, R. Moessner, and P. Zoller, "Quantum spin-ice and dimer models with rydberg atoms," Phys. Rev. X 4, 041037 (2014).

[26] A. W. Glaetzle, M. Dalmonte, R. Nath, C. Gross, I. Bloch, and P. Zoller, "Designing frustrated quantum magnets with laser-dressed rydberg atoms," Phys. Rev. Lett. 114, 173002 (2015).

[27] T. E. Lee, H. Häffner, and M. C. Cross, "Antiferromagnetic phase transition in a nonequilibrium lattice of rydberg atoms," Phys. Rev. A 84, 031402 (2011).

[28] T. E. Lee, S. Gopalakrishnan, and M. D. Lukin, "Unconventional magnetism via optical pumping of interacting spin systems," Phys. Rev. Lett. 110, 257204 (2013). 
[29] A. Neuzner, M. Körber, O. Morin, S. Ritter, and G. Rempe, "Interference and dynamics of light from a distance-controlled atom pair in an optical cavity,” Nat Photon 10, 303 (2016).

[30] J. Ningyuan, A. Georgakopoulos, A. Ryou, N. Schine, A. Sommer, and J. Simon, “Observation and characterization of cavity rydberg polaritons," Phys. Rev. A 93, 041802 (2016).

[31] M. Litinskaya, E. Tignone, and G. Pupillo, "Cavity polaritons with rydberg blockade and long-range interactions," arXiv:1607.08323 (2016).

[32] D. Maxwell, D. J. Szwer, D. Paredes-Barato, H. Busche, J. D. Pritchard, A. Gauguet, K. J. Weatherill, M. P. A. Jones, and C. S. Adams, "Storage and control of optical photons using rydberg polaritons," Phys. Rev. Lett. 110, 103001 (2013).

[33] C. Tresp, P. Bienias, S. Weber, H. Gorniaczyk, I. Mirgorodskiy, H. P. Büchler, and S. Hofferberth, “Dipolar dephasing of rydberg $d$-state polaritons,” Phys. Rev. Lett. 115, 083602 (2015).

[34] G. Sagué, E. Vetsch, W. Alt, D. Meschede, and A. Rauschenbeutel, "Cold-atom physics using ultrathin optical fibers: Light-induced dipole forces and surface interactions,” Phys. Rev. Lett. 99, 163602 (2007).

[35] J. D. Thompson, T. G. Tiecke, N. P. de Leon, J. Feist, A. V. Akimov, M. Gullans, A. S. Zibrov, V. Vuletić, and M. D. Lukin, "Coupling a single trapped atom to a nanoscale optical cavity," Science 340, 1202 (2013).

[36] J. Petersen, J. Volz, and A. Rauschenbeutel, "Chiral nanophotonic waveguide interface based on spin-orbit interaction of light," Science 346, 67 (2014).

[37] R. Boddeda, I. Usmani, E. Bimbard, A. Grankin, A. Ourjoumtsev, E. Brion, and P. Grangier, "Rydberg-induced optical nonlinearities from a cold atomic ensemble trapped inside a cavity," Journal of Physics B: Atomic, Molecular and Optical Physics 49, 084005 (2016).

[38] R. J. Bettles, S. A. Gardiner, and C. S. Adams, "Cooperative ordering in lattices of interacting twolevel dipoles,” Phys. Rev. A 92, 063822 (2015).

[39] M. Hoening, W. Abdussalam, M. Fleischhauer, and T. Pohl, "Antiferromagnetic long-range order in dissipative rydberg lattices," Phys. Rev. A 90, 021603 (2014).

[40] F. Dimer, B. Estienne, A. S. Parkins, and H. J. Carmichael, "Proposed realization of the dicke-model quantum phase transition in an optical cavity qed system,” Phys. Rev. A 75, 013804 (2007).

[41] N. Dogra, F. Brennecke, S. D. Huber, and D. T., "Phase transitions in a bose hubbard model with cavity-mediated global-range interactions," arXiv:1604.00865 (2016). 
[42] Y. Chen, Z. Yu, and H. Zhai, "Quantum phase transitions of the bose-hubbard model inside a cavity," Phys. Rev. A 93, 041601 (2016).

[43] Y. Li, L. He, and W. Hofstetter, "Lattice-supersolid phase of strongly correlated bosons in an optical cavity," Phys. Rev. A 87, 051604 (2013).

[44] K. Baumann, C. Guerlin, F. Brennecke, and T. Esslinger, "Dicke quantum phase transition with a superfluid gas in an optical cavity," Nature 464, 1301 (2010).

[45] M. P. Baden, K. J. Arnold, A. L. Grimsmo, S. Parkins, and M. D. Barrett, "Realization of the dicke model using cavity-assisted raman transitions,” Phys. Rev. Lett. 113, 020408 (2014).

[46] J. Gelhausen, M. Buchhold, and P. Strack, "Many-body quantum optics with decaying atomic spin states," arXiv:1605.07637 (2016).

[47] P. Domokos and H. Ritsch, "Collective cooling and self-organization of atoms in a cavity," Phys. Rev. Lett. 89, 253003 (2002).

[48] A. T. Black, H. W. Chan, and V. Vuletić, "Observation of collective friction forces due to spatial self-organization of atoms: From rayleigh to bragg scattering,” Phys. Rev. Lett. 91, 203001 (2003).

[49] T. Senthil, A. Vishwanath, L. Balents, S. Sachdev, and M. P. A. Fisher, "Deconfined quantum critical points," Science 303, 1490 (2004).

[50] R. Mottl, F. Brennecke, K. Baumann, R. Landig, T. Donner, and T. Esslinger, "Roton-type mode softening in a quantum gas with cavity-mediated long-range interactions," Science 336, 1570 (2012).

[51] F. Brennecke, R. Mottl, K. Baumann, R. Landig, T. Donner, and T. Esslinger, "Real-time observation of fluctuations at the driven-dissipative dicke phase transition," PNAS 110, 11763 (2013).

[52] F. Piazza and P. Strack, "Quantum kinetics of ultracold fermions coupled to an optical resonator," Phys. Rev. A 90, 043823 (2014).

[53] M. Kulkarni, B. Öztop, and H. E. Türeci, "Cavity-mediated near-critical dissipative dynamics of a driven condensate," Phys. Rev. Lett. 111, 220408 (2013).

[54] G. Kónya, G. Szirmai, and P. Domokos, "Damping of quasiparticles in a bose-einstein condensate coupled to an optical cavity," Phys. Rev. A 90, 013623 (2014).

[55] R. J. Thompson, G. Rempe, and H. J. Kimble, "Observation of normal-mode splitting for an atom in an optical cavity,” Phys. Rev. Lett. 68, 1132 (1992).

[56] E. G. D. Torre, S. Diehl, M. D. Lukin, S. Sachdev, and P. Strack, "Keldysh approach for nonequilibrium phase transitions in quantum optics: Beyond the dicke model in optical cavities," Phys. Rev. A 87, 023831 (2013). 
[57] M. H. Szymanska, J. Keeling, and P. B. Littlewood, "Nonequilibrium quantum condensation in an incoherently pumped dissipative system," Phys. Rev. Lett. 96, 230602 (2006).

[58] M. H. Szymańska, J. Keeling, and P. B. Littlewood, "Mean-field theory and fluctuation spectrum of a pumped decaying bose-fermi system across the quantum condensation transition,’ Phys. Rev. B 75, 195331 (2007).

[59] S. Schütz and G. Morigi, "Prethermalization of atoms due to photon-mediated long-range interactions,” Phys. Rev. Lett. 113, 203002 (2014).

[60] M. J. Bhaseen, J. Mayoh, B. D. Simons, and J. Keeling, "Dynamics of nonequilibrium dicke models," Phys. Rev. A 85, 013817 (2012).

[61] T. E. Lee and M. C. Cross, "Quantum-classical transition of correlations of two coupled cavities," Phys. Rev. A 88, 013834 (2013).

[62] J. Honer, H. Weimer, T. Pfau, and H. P. Büchler, "Collective many-body interaction in rydberg dressed atoms," Phys. Rev. Lett. 105, 160404 (2010).

[63] G. Pupillo, A. Micheli, M. Boninsegni, I. Lesanovsky, and P. Zoller, "Strongly correlated gases of rydberg-dressed atoms: Quantum and classical dynamics," Phys. Rev. Lett. 104, 223002 (2010).

[64] N. Henkel, R. Nath, and T. Pohl, "Three-dimensional roton excitations and supersolid formation in rydberg-excited bose-einstein condensates,” Phys. Rev. Lett. 104, 195302 (2010).

[65] A. W. Glaetzle, R. Nath, B. Zhao, G. Pupillo, and P. Zoller, "Driven-dissipative dynamics of a strongly interacting rydberg gas," Phys. Rev. A 86, 043403 (2012).

[66] F. Nogrette, H. Labuhn, S. Ravets, D. Barredo, L. Béguin, A. Vernier, T. Lahaye, and A. Browaeys, "Single-atom trapping in holographic $2 \mathrm{~d}$ arrays of microtraps with arbitrary geometries," Phys. Rev. X 4, 021034 (2014).

[67] M. J. Collett and C. W. Gardiner, "Squeezing of intracavity and traveling-wave light fields produced in parametric amplification,” Phys. Rev. A 30, 1386 (1984).

[68] C. W. Gardiner and M. J. Collett, "Input and output in damped quantum systems: Quantum stochastic differential equations and the master equation,” Phys. Rev. A 31, 3761 (1985).

[69] E. G. Dalla Torre, E. Demler, T. Giamarchi, and E. Altman, "Dynamics and universality in noisedriven dissipative systems," Phys. Rev. B 85, 184302 (2012).

[70] T. E. Lee, H. Häffner, and M. C. Cross, "Collective quantum jumps of rydberg atoms," Phys. Rev. Lett. 108, 023602 (2012). 
[71] P. Schad, Y. Makhlin, B. Narozhny, G. Schoen, and A. Shnirman, "Majorana representation for dissipative spin systems," Annals of Physics 361, 401 (2015).

[72] M. Babadi, E. Demler, and M. Knap, "Far-from-equilibrium field theory of many-body quantum spin systems: Prethermalization and relaxation of spin spiral states in three dimensions," Phys. Rev. X 5, 041005 (2015).

[73] D. E. Chang, J. I. Cirac, and H. J. Kimble, "Self-organization of atoms along a nanophotonic waveguide,” Phys. Rev. Lett. 110, 113606 (2013).

[74] T. Ramos, H. Pichler, A. J. Daley, and P. Zoller, "Quantum spin dimers from chiral dissipation in cold-atom chains," Phys. Rev. Lett. 113, 237203 (2014).

[75] H. Pichler, T. Ramos, A. J. Daley, and P. Zoller, "Quantum optics of chiral spin networks," Phys. Rev. A 91, 042116 (2015).

[76] A. Asenjo-Garcia, J. D. Hood, D. E. Chang, and H. J. Kimble, "Atom-light interactions in a quasi-1d nanostructures: a green's function perspective," arXiv:1606.04977 (2016).

[77] J. S. Douglas, H. Habibian, C. L. Hung, A. V. Gorshkov, H. J. Kimble, and D. E. Chang, "Quantum many-body models with cold atoms coupled to photonic crystals," Nat Photon 9, 326 (2015).

[78] J. Thompson and M. Endres, "Quantum simulation: Choose your own interaction,” Nat Photon 9, 285 (2015).

[79] J. Schmitt, T. Damm, D. Dung, F. Vewinger, J. Klaers, and M. Weitz, "Thermalization kinetics of light: From laser dynamics to equilibrium condensation of photons," Phys. Rev. A 92, 011602 (2015).

[80] A. J. Kollar, A. T. Papageorge, V. D. Vaidya, Y. Guo, J. Keeling, and B. L. Lev, "Supermode-densitywave-polariton condensation," arXiv:1606.04127 (2016). 